\title{
Proceedings of the Second Neurological Disorders Summit (NDS-2016)
}

\section{$\underline{\text { Keynote Presentations }}$}

\section{Progressive Autophagy-lysosomal Deficits Contribute to fALS-linked Motor Neuron Degeneration}

\author{
Zu-Hang Sheng \\ National Institute of Neurological Disorders and Stroke, NIH, MD, USA
}

\begin{abstract}
One pathological hallmark in ALS-linked motor neurons (MNs) is axonal accumulation of damaged mitochondria, which produce energy and buffer $\mathrm{Ca}^{2+}$ less efficiently, and initiate apoptotic cascades and axonal degeneration. These observations raise a fundamental question: Does impaired removal of those damaged mitochondria by the autophagy-lysosome system play a pathological role during the early asymptomatic stage of fALS-linked mice? We recently reveal for the first time spinal MN-targeted progressive lysosomal deficits starting at asymptomatic stages in fALS-linked hSOD1G93A mice. These deficits impair autophagic degradation, resulting in aberrant accumulation of autophagic vacuoles engulfing damaged mitochondria along $\mathrm{MN}$ axons. These phenotypes were captured in cultured adult (P40) spinal MNs from the hSOD1G93A mice. Such early deficits are due to reduced late endosome (LE) retrograde transport via binding of mutant hSOD1G93A to dynein and can be reversed by introducing dynein adaptor snapin transgene. Snapin competes with hSOD1G93A for binding to dynein, thereby recruiting more dynein to LEs for transport. Thus, snapin and hSOD1G93A play opposite roles in LE retrograde transport. Expressing snapin efficiently reverses lysosome deficits and facilitates removal of damaged mitochondria. Injecting AAV9-snapin into the diseased mice rescues lysosome deficits and slows MN degeneration in vivo and disease progression. Our study advances our knowledge of early pathological mechanisms underlying MN degeneration. Enhancing clearance of damaged mitochondria by regulating endolysosomal trafficking may be a potential therapeutic strategy for ALS and perhaps other neurodegenerative diseases. (Supported by the Intramural Research Program of NINDS, NIH).
\end{abstract}

\section{Synesthesia and the Creative Spark}

\author{
Richard E. Cytowic \\ George Washington University, USA
}

\begin{abstract}
The under-and misdiagnosed trait of synesthesia- coupled sensations- concerns all neurologists whose council affected individuals or their parents often seek. Sharing a root with anesthesia ("no sensation"), synesthesia ("coupled sensation") affects $4 \%$ of the population-far more than Parkinson's disease, epilepsy, or typical conditions that neurologists see. Synesthesia is highly hereditable but has incomplete penetrance, leaving 1 in 90 individuals with some kind of overt perceptual coupling-still an enormous number open to being misunderstood. Common manifestations are colored and personified alphabets, words that have distinct tastes, three dimensional "number forms" for overlearned sequences, and sounds such as music and voices that produce a firework-like display of moving, colored shapes. 35 years ago, Richard Cytowic began to nudge this curious oddity back to mainstream science, which had all but forgotten its 200-year history. First dismissed as New Age weirdness, synesthesia's reality caused a paradigm shift: it is now seen as fundamental to understanding how all brains perceive. Nowhere is the trait more important than in understanding metaphoric thinking. Whether one is overtly synesthetic like Kandinsky, Nabokov, Lady
\end{abstract}


Gaga, or not, the capacity to see the similar in the dissimilar is the mark of metaphoric thinking, embodied perception, and creative insight. Synesthesia also poses severe challenges to efforts at artificial intelligence and brain-inspired computing such as IBM's Watson and its True North neuromorphic chip.

\title{
At the Crossroads Between Promotion of Health and Prevention of Age-related Neurodegeneration with Natural Drugs to Avoid the Catastrophic Cliff of Neuronal Failure
}

\author{
Giulio Maria Pasinetti \\ Icahn School of Medicine at Mount Sinai, New York, NY, USA
}

\begin{abstract}
Polyphenols are a large and diverse group of naturally occurring compounds widely distributed in many plant-derived foods and beverages. Polyphenols are receiving increasing attention for their potential role in the promotion of resilience in response to stress-induced psychological impairment and cognitive deterioration. In particular, preclinical evidence demonstrated the efficacy of certain polyphenols, acting either individually or in combination, to modulate multiple diverse mechanisms relevant to depression and anxiety, which also pose a risk to neurodegeneration such as Alzheimer's disease, implicating the potential for novel development of polyphenols for multi-target engagement. In spite of the increasing efforts committed to clinical testing of polyphenols, these efforts are hindered by limited knowledge of polyphenol bioavailability, specific forms of brainbioavailable bioactive polyphenols (including polyphenol metabolites) and their underlying mechanisms of actions. The overall goal of this presentation is to provide an overview on the "state of the art" on the development of polyphenols and eventually their translation into the clinical setting. In particular, I will discuss the mechanistic implications of brain-bioavailable bioactive polyphenol metabolites in modulating key physiological processes that are relevant in preventative and therapeutic approaches to stress-induced psychological impairment and cognitive deterioration. Moreover, emerging evidence suggests that there is a pivotal role of the gastrointestinal microbiota in mechanisms associated with bioavailability of bioactive polyphenol metabolites. Based on this consideration, I will discuss novel evidence about the role of microbiota in modulating the bioavailability of bioactive phenolic acids from polyphenols. Collectively, in my presentation, I will critically discuss the current complex mosaic of evidence, which puts the investigation of brain-bioavailable bioactive polyphenols at the cross-roads between promotion of health and prevention of age-related psychological resilience and prevention of age related degenerative disorders.
\end{abstract}

\section{Opioid Reward Mechanisms: A Potential Role in Metabolic Disturbances?}

\author{
Igor Elman \\ Boonshoft School of Medicine, Wright State University and Dayton VA Medical Center, OH, USA
}

\begin{abstract}
Obesity and overeating are viewed by some as type of addiction, but the underlying mechanisms remain unclear. Central opioidergic system may play a role as evidenced by its: a) enhancement of rewarding features of food; b) boosting orexigenic and suppressing anorexigenic neuropeptides; c) reducing peripheral insulin secretion and d) desensitizing insulin receptors. The purpose of this presentation is to discuss a heuristic value of opioid blockade for patients' metabolic status. First, four groups of Wistar Han IGS rats were treated with a second generation antipsychotic, olanzapine, a combination of olanzapine and an opioid receptor antagonist, naltrexone, naltrexone alone or vehicle. Second, patients with heroin dependence underwent the sweet taste test before- and after the injection of depot naltrexone. Third, we conducted a double-blind placebo-controlled pilot clinical trial where schizophrenic patients on a stable dose of olanzapine were randomized in a double-blind fashion to receive naltrexone or placebo. Rats treated with olanzapine and naltrexone were similar to the vehicle-treated animals with respect to food intake and body weight gain, whereas olanzapine treatment alone induced overeating and obesity. Data from heroin dependent human subjects demonstrated a reduction in the hedonic and motivational ratings of sweet solutions after naltrexone. On the clinical trial, in comparison to the olanzapine and placebo combination, the olanzapine and naltrexone group displayed a significant decrease in the fat mass, assessed with the biometric impedance analysis and a trend towards improvement in the insulin resistance quantified via HOMA-IR values. In conclusion, naltrexone addition may result in clinically meaningful attenuation of metabolic problems. Potential mechanisms of naltrexone action may involve diminution of rewarding features of food in conjunction with favorable effects on insulin sensitivity.
\end{abstract}




\title{
First Case of Brain Protection in Out of Hospital Cardiac Arrest
}

\author{
Guy Hugues Fontaine \\ Hôpital de la Salpêtrière and Université Pierre et Marie Curie, Paris, France
}

\begin{abstract}
The case of my wife Ilfat, a 73-year-old psychiatrist, experienced OHCA on June 2011 in the living room where she was watching the BBC world channel on TV. She suddenly stopped talking. I waited a few moments before turning towards her to check whether she might have fallen asleep. To my horror I found that she was cyanotic, her head drooped on her chest. It was obvious that she had signs of possible sudden cardiac arrest. I immediately laid her down on the floor. There was no femoral pulse. I initiated standard CPR including cardiac massage (90 compressions/minute) for about 30 seconds followed by mouth to mouth breathing. I then resumed cardiac massage since she did not regain consciousness and there was no femoral pulse. I had a defibrillator in the basement of my house because of my interest in the Fulguration procedure. Although the defibrillator was more than 30 years old I knew that this machine would work by electrical current from the wall circuit. The defibrillator was charged to allow for a first shock to be delivered through metallic disk-shaped electrodes that were on the old machine. Her body jumped but the shock was ineffective. Since I had no gel to apply between the electrodes and the skin to decrease the impedance, I applied saliva to the electrodes and then gave a second and third shock. This last shock was successful, and the femoral pulse returned. Recurrent chest compression produced the characteristic sound make by a broken chondro-costal joint. The femoral pulse remained stable, regular and strong. I estimated that about 5 to 6 minutes elapsed between her loss of consciousness and the return to a stable circulation. Since there was no sign of return to consciousness and she had bilateral pupillary dilation, I then addressed the issue of possible brain protection. Fortunately, I had a smaller portable cylinder that was designed with a gas regulator to be used with a nasal cannula inserted into the nose to provide protection of the brain by nasal cooling.
\end{abstract}

Because of the invasive insertion, Ilfat exhibited a pain reaction which I interpreted as a positive sign for her chances of survival. When the injector was in position I opened the valve of the cylinder and induced nasal cooling by expansion of the $\mathrm{CO}_{2}$. Her hospital course was notable for repeated episodes of ventricular fibrillation. When sedation was stopped she was fully awake and the tracheal tube was removed. Ilfat first question was whether she was treated with nasal cooling! She was treated with amiodarone in combination with bisoprolol which proved effective. After recovery from the implantation of the defibrillator she had only thoracic pain which was a direct consequence of chest compression. This pain disappeared in two months. Psychological test showed that she had absolutely no neurologic deficit. In particular, she was able to remember 9 telephone numbers.

\section{Hypothesizing that Neuropharmacological and Neuroimaging Studies of Glutaminergic-Dopaminergic Optimization Complex (KB220Z) are Indicative of Producing “Dopamine Homeostasis” in Reward Deficiency Syndrome (RDS)}

\author{
Kenneth Blum \\ Keck School of Medicine of USC, Los Angeles, CA, USA
}

\begin{abstract}
There is a need to find better treatments for all addictive behaviors, both substance and non-substance related, termed Reward Deficiency Syndrome (RDS). While the FDA has approved a number of powerful pharmaceuticals under the umbrella term Medication Assisted Treatment (MAT), these drugs are not optimal. It is our contention that these drugs work well in the short-term by blocking dopamine function that leads to a psychological extinction. However, especially for the opiate/ opioid dependent individuals, the standard use of buprenorphine/naloxone used over a long period of time results in unwanted addiction liability, reduced affect, and mood changes including suicidal ideation. My group hereby is proposing a paradigm shift in the treatment arena, whereby the long-term goal should embrace "Dopamine Homeostasis."While this is a laudable goal, it is very difficult to achieve. However, this keynote presentation briefly reviews some of the past history of utilizing a glutaminergicdopaminergic optimization complex [KB220Z] shown to be beneficial in at least 25 clinical trials and number of published and unpublished studies. It is our group's opinion that, while additional required studies could confirm these findings to date, these cited studies are indicative of achieving enhanced resting state functional connectivity, connectivity volume, and even possibly, neuroplasticity. We are proposing a Reward Deficiency Solution System (RDSS) that includes: Genetic Addiction Risk Score (GARS); Comprehensive Analysis of Reported Drugs (CARD); and a glutaminergic-dopaminergic optimization complex (KB220Z). Continued investigation of this novel strategy may lead to a better-targeted approach in the long-term causing
\end{abstract}


dopamine regulation by balancing the glutaminergic-dopaminergic pathways. This will potentially change the landscape of treating all addictions leading us to the promised land.

\title{
Study of Dysregulated Neurotransmission Using Dynamic Molecular Imaging Technique
}

\author{
Rajendra D. Badgaiyan \\ Department of Psychiatry, University of Minnesota, Minneapolis, MN, USA
}

\begin{abstract}
Neuroimaging techniques have extremely limited ability to detect task-induced acute changes in the levels of neurotransmitters. As a result, we have limited understanding of the pathophysiology of psychiatric and neuropsychiatric conditions. To address this limitation, we developed the Single-scan Dynamic Molecular Imaging Technique (SDMIT). It allows detection, mapping and measurement of dopamine released acutely during performance of a cognitive or behavioral task in the live human brain. The technique uses models to measure values of the receptor kinetic parameters. We used the technique to to resolve controversy concerning status of dopamine neurotransmission in attention deficit hyperactivity disorder (ADHD). Studies that have used indirect methods to measure dopamine neurotransmission have reported either increased, decreased, or unchanged levels of dopaminergic activity in ADHD. The contradictory findings could be due to inability of these methods to differential phasic and tonic release of dopamine. Using SDMIT we found that dopamine release at rest (tonic release) was significantly lower in ADHD patients than that in healthy control volunteers in the right caudate. Further, the phasic release during performance of a Flanker Task was significantly higher in ADHD volunteers in the same area. These results help to resolve the controversy concerning pathophysiology of ADHD. Further, the experiment demonstrates that SDMIT can be used to study pathophysiology of psychiatric and neuropsychiatric conditions that are associated with dysregulated neurotransmission.
\end{abstract}




\title{
Featured Presentations
}

\section{Challenges in Neurologic Drug Development}

\author{
Mohamed El Mouelhi \\ Novartis Institutes for Biomedical Research, MA, USA
}

\begin{abstract}
Drug development process appeared to be a black box for the majority of people questioning the prescription high cost. The process to develop new drugs is lengthy and costly. Phases of drug development will be described. Only one out of 10 compounds entering first study in man (Phase 1) reaches the market after an average of 14 years with a cost of $\$ 2.7$ billion. As reported by the Centers for Medicare and Medicaid services, prescription drugs constituted only $9 \%$ of each health care dollar spent in US in 2013. The many challenges encountered in neurological clinical trials will be discussed. The use of social media and risk of bias is a specific challenge introduced by recent technology that would interfere with accurate interpretation of collected data. This risk needs to be minimized. The concept of collaborative work between regulators, sponsors and patient advocacy groups should prevail and lead to achievement of their common goal that is having effective and safe treatment available for all patients.
\end{abstract}

\section{AtheroCloud ${ }^{\mathrm{TM}}$ : A Web-based Point-of-care Solution for Accurate IMT Measurement System for Stroke Risk Monitoring}

\author{
Luca Saba $^{1}$, Sumit K. Banchhor ${ }^{2}$, Tadashi Araki ${ }^{3}$, Nobutaka Ikeda ${ }^{4}$, Narendra D. Londhe ${ }^{2}$, Klaudija Viskovic ${ }^{5}$, Shoaib \\ Shafique $^{6}$, Ajay Gupta ${ }^{7}$, Andrew Nicolaides ${ }^{8,9}$, John R. Laird ${ }^{10}$ and Jasjit S. Suri ${ }^{11-13^{*}}$
}

${ }^{1}$ Department of Radiology, University of Cagliari, Italy

${ }^{2}$ Department of Electrical Engineering, NIT Raipur, Chbattisgarh, India

${ }^{3}$ Division of Cardiovascular Medicine, Toho University Ohashi Medical Center, Tokyo, Japan

${ }^{4}$ Cardiovascular Medicine, National Center for Global Health and Medicine, Tokyo, Japan

${ }^{5}$ Department of Radiology and Ultrasound, University Hospital for Infectious Disease, Zagreb, Croatia

${ }^{6}$ CorVasc Vascular Laboratory, 8433 Harcourt Rd \#100, Indianapolis, IN, USA

${ }^{7}$ Brain and Mind Research Institute, Weill Cornell Medical College, NY, USA

${ }^{8}$ Vascular Screening and Diagnostic Centre, London, England

${ }^{9}$ Vascular Diagnostic Centre, University of Cyprus, Nicosia, Cyprus

${ }^{10}$ UC Davis Vascular Centre, University of California, Davis, CA, USA

${ }^{11}$ Monitoring and Diagnostic Division, AtheroPoint ${ }^{T M}$, Roseville, CA, USA

${ }^{12}$ Point-of-Care Devices, Global Biomedical Technologies, Inc., Roseville, CA, USA

${ }^{13}$ Department of Electrical Engineering, University of Idaho (Affl.), ID, USA

\begin{abstract}
This study presents AtheroCloud ${ }^{\mathrm{TM}}$ - a novel cloud-based smart carotid intima-media thickness (cIMT) measurement using B-mode ultrasound for stroke risk assessment and monitoring. The ultrasound arterial scans can be uploaded from the local server into the Cloud and cIMT measurements can be computed by multiple users around the globe. Benchmarking of AtheroCloud ${ }^{\mathrm{TM}}$ was done against Sonographer and manual tracings. Further, the system was tested for inter- and intra-observer variability analysis. One hundred patients ( $75 \mathrm{M} / 25 \mathrm{~F}$, mean age: $68 \pm 11$ years), IRB approved, Toho University, Japan, consisted of Left/Right CCA artery (200 ultrasound images), (Toshiba, Tokyo, Japan) were collected using a 7.5-MHz transducer. The coefficient of correlation (CC) between Sonographer and manual readings for Left/Right cIMT was $0.74(\mathrm{P}<0.0001)$ and $0.65(\mathrm{P}<0.0001)$, while, between AtheroCloud ${ }^{\mathrm{TM}}$ and manual was $0.96(\mathrm{P}<0.0001)$ and $0.97(\mathrm{P}<0.0001)$, respectively. We observed that $91.15 \%$ of the population in AtheroCloud ${ }^{\mathrm{TM}}$ had a mean cIMT error less than $0.11 \mathrm{~mm}$, compared to $61 \%$ for Sonographer's reading. The AUC for the ROC was 0.99 for AtheroCloud ${ }^{\mathrm{TM}}$ compared to 0.81 for Sonographer. The mean $\mathrm{CC}$ for intra-operator and inter-operator reproducibility between all the three automated reading pairs was $0.99(\mathrm{P}<0.0001)$ and $[0.96(\mathrm{P}<0.0001)$, respectively. The CC for intra-observer variability between manual-1 and manual-2 readings was 0.92 $(\mathrm{P}<0.0001)$ and $0.97(\mathrm{P}<0.0001)$, respectively. The AtheroCloud ${ }^{\mathrm{TM}}$ system showed very high reproducibility and negligible intra/inter-observer variability. Statistical tests were performed to demonstrate consistency, reliability and accuracy of the system. The proposed AtheroCloud ${ }^{\mathrm{TM}}$ system is completely automated, fast, accurate, reproducible, reliable, anytime-anywhere and smart tool for multi-center clinical trials and routine vascular screening.
\end{abstract}




\title{
Molecular Mediators, Environmental Modulators and Epigenetic Mechanisms in Mouse Models of Brain Disorders
}

\author{
Burrows $^{1}$, Thibault Renoir ${ }^{1}$, Terence Y. Pang ${ }^{1}$ and AnthonyJ. Hannan ${ }^{1,4^{*}}$ \\ ${ }^{1}$ Florey Institute of Neuroscience and Mental Health, University of Melbourne, Australia \\ ${ }^{2}$ Department of Neurobiology, University of Chicago, IL, USA \\ ${ }^{3}$ University of California, Irvine, CA, USA \\ ${ }^{4}$ Department of Anatomy and Neuroscience, University of Melbourne, Australia
}

Annabel K. Short ${ }^{1,2}$, Shlomo Yeshurun ${ }^{1}$, Katie A. Fennell ${ }^{1}$, Dean J. Wright ${ }^{1}$, Christina Mo ${ }^{1,2}$, Shanshan Li ${ }^{1}$, Emma L.

\begin{abstract}
Huntington's disease (HD) is a tandem repeat expansion disorder involving psychiatric, cognitive and motor symptoms. In a transgenic mouse model of $\mathrm{HD}$ we have discovered that environmental enrichment can delay disease onset. We have also provided the first evidence that chronic stress, as well as elevated stress hormone alone, can accelerate the onset of disease in HD. In particular, we demonstrated that increased corticosterone (CORT) levels accelerated the onset of cognitive dysfunction, modelling dementia, in HD mice. Increased CORT levels also accelerated onset of cellular plasticity and molecular deficits in specific brain regions of the HD mice. Our results suggest that the timing and duration of these environmental manipulations are critical in terms of their ability to modify gene expression. Furthermore, we have used our molecular discoveries to inform preclinical pharmacological studies. Together with epidemiological studies and clinical trials, this research is informing the design of ongoing intervention studies for HD. We have also explored the transgenerational effects of paternal stress. To investigate how paternal stress might influence offspring behavioral phenotypes, a model of hypothalamic-pituitary-adrenal (HPA) axis dysregulation in male C57/B16 mice was used. Paternal drinking water was supplemented with oral CORT before mating with untreated females. Offspring were then tested for a variety of behavioural, cellular and molecular assays. Our findings reveal significant effects on offspring via transgenerational epigenetic inheritance. Sustained elevation of glucocorticoids is therefore instrumental in the transmission of paternal stress-induced traits across generations and occurs via specific epigenetic modifications in the sperm of stressed fathers.
\end{abstract}

\section{Brain Protection by Adiabatic $\mathrm{CO}_{2}$ Expansion for Out of Hospital Cardiac Arrest, Stroke and Spinal Cord Injury on Animal Models and First Fortuitous Clinical Case}

\section{Fontaine Guy Hugues}

Hopital de La Salpêtrière and Pierre et Marie Curie University, School of Medicine, Paris, France

\begin{abstract}
Therapeutic hypothermia produced by evaporation of Per Fluoro Carbon in the fossa nasalis in a flow of oxygen reported in a prospective multicentre study has demonstrated a tendency to improve outcome in out of hospital cardiac arrest. When I saw the experiments on pigs at the Weil Institute of Cardiac Care Medicine (WICCM) I was immediately convinced that another approach of cooling could be abrupt decompression of gas. After multiple experiments I demonstrated that it was possible to obtain on the same model a drop of brain temperature similar to the work previously reported at the WICCM. However, the simultaneous work on infrared images on severed pig heads suggested that it was possible to cool the brain by the decompression of gas inside the mouth instead of the nose which looks to be an even less invasive and faster method on the field. The second major interest of this new technique seems to be its application in stroke also suggested by animal models. It was during these experiments that my wife watching TV beside me experienced an episode of cardiac arrest that I was able to diagnose immediately. I started Cardio Pulmonary Resuscitation followed by defibrillation performed by an old but still working defibrillator that I kept in the basement of my house when the original study of the method of fulguration was completed. Before arrival of Fire Brigade I used a bottle of compressed gas also available in the basement of my house and delivered the cooling gas at that time in the fossa nasalis. Despite a period of 6 min of no-flow she was able to recover after 5 days of coma with absolutely no neurologic deficit. This fortuitous resuscitation using for the first time brain cooling by decompressed gas will be used for the first pilot study supported by the Schiller Company (Switzerland). It will start soon in the city of Lugano which is already famous for its highest success rate of resuscitation (50\%).
\end{abstract}




\title{
Prospective Early Biomarkers for Alzheimer's Disease Based on Cell Cycle Alterations in Blood Lymphocytes
}

\author{
Urszula Wojda*, Joanna Wojsiat, Katarzyna Laskowska-Kaszub and Anna Mietelska-Porowska \\ Laboratory of Preclinical Testing of Higher Standards, Nencki Institute of Experimental Biology, Warsaw, Poland
}

\begin{abstract}
Alzheimer's disease $(\mathrm{AD})$ is a progressive, irreversible neurodegenerative disorder and the most common age-related dementia generating an overwhelming socioeconomic burden on society. Mounting evidence indicates that the effectiveness of therapeutic modalities critically depends on the early diagnosis of AD. Unfortunately, no preclinical diagnostic methods exist and available treatments for AD are only symptomatic. It becomes clear that progress in AD therapy and diagnostics relies to a great extent on the elucidation of early molecular AD pathomechanisms, and the identification of novel drug targets and biomarkers of early AD, preferably in easily available diagnostic materials, such as blood. Our recent studies clearly demonstrated aberrant regulation of the cell cycle in peripheral lymphocytes in $\mathrm{AD}$, similarly as reported previously in brain neurons. Real-time PCRarrays, immunoblotting, and flow cytometry demonstrated differences in the regulation of G1/S phases between sporadic AD lymphocytes and cells from age-matched non-demented subjects. Thus, research on lymphocytes showed the systemic nature of the basic cellular process changed in AD. Moreover, similar dysfunctions were demonstrated in lymphocytes of mild cognitive impairment patients (MCI), which suggests that these are early defects in AD. These findings provide support for the cell cycle hypothesis of $\mathrm{AD}$ pathogenesis and open perspectives for the application of easily accessible blood lymphocytes for the development of new diagnostic methodologies involving cell cycle-based early AD biomarkers.
\end{abstract}

\section{Olfactory Dysfunction in Parkinson's and Other Neurological Diseases: The Search for a Common Pathological Substrate}

\author{
Richard L. Doty \\ Perelman School of Medicine, University of Pennsylvania, Philadelphia, PA, USA
}

\begin{abstract}
The sense of smell, i.e. Cranial Nerve I, is frequently overlooked and rarely tested by neurologists and other medical practitioners. This is in spite of its importance to the patient and the fact that olfactory dysfunction is an early biomarker of a number of neurological diseases, most notably Parkinson's disease (PD) and Alzheimer's disease (AD). In contrast to these two diseases, such neurodegenerative diseases as progressive supranuclear palsy (PSP), amyotrophic lateral sclerosis (ALS), and essential tremor (ET) have comparatively little olfactory dysfunction, making olfactory testing useful in differential diagnosis. Given the marked similarity between the olfactory losses observed in AD, PD, and such seemingly unrelated diseases as Korsakoff psychosis, myasthenia gravis, and Chaga's disease, the question arises as to whether a common neuropathological substrate underlies the olfactory disturbances. If this is the case, does olfactory dysfunction precede the development of the pathognomonic markers of such diseases as $\mathrm{PD}$ and $\mathrm{AD}$, potentially exposing yet-to-be-defined disease-related precursors of the defining pathology? Do the harbingers subsequently become correlated with or confounded by the classic pathology, thereby confusing the issue of primary cause and effect? In this presentation I explore these issues and pose the hypothesis that damage to the forebrain cholinergic system, in some cases caused or exacerbated by innate immune responses, may well explain most of the similarities and differences in olfactory function among a wide range of seemingly disparate neurological diseases.
\end{abstract}

\section{Interneuron Transcriptional Dysregulation Causes Altered Modulation by Haloperidol of Inhibitory Synaptic Transmission in Hippocampus}

\author{
Aundrea F. Bartley ${ }^{1}$, Sarah E. Dougherty ${ }^{2}$, Elizabeth K. Lucas ${ }^{2}$, Lillian B. Brady ${ }^{1}$, Qin Li ${ }^{1}$,John J. Hablitz ${ }^{1}$, Rita M. Cowell ${ }^{2}$ \\ and Lynn E. Dobrunz ${ }^{*}$ \\ ${ }^{1}$ Department of Neurobiology, Evelyn F. McKnight Brain Institute, and Civitan International Research Center, University of Alabama, \\ Birmingham, Alabama, AL, USA \\ ${ }^{2}$ Department of Psychiatry and Behavioral Neurobiology, and Civitan International Research Center, University of Alabama, \\ Birmingham, Alabama, AL, USA
}




\begin{abstract}
Peroxisome proliferated-activated receptor $\gamma$ coactivator $1 \alpha(\mathrm{PGC}-1 \alpha)$ is a transcriptional coactivator implicated in a wide range of neurological disorders, including Parkinson Disease, Alzheimer Disease, Huntington Disease, schizophrenia, and anxiety disorders. PGC-1 $\alpha$ is known to regulate metabolic genes, both in neurons and in non-neuronal cells. In the central nervous system, PGC- $1 \alpha$ is highly concentrated in inhibitory interneurons, and its deletion leads to reduced levels of the calcium binding protein parvalbumin. In addition, PGC- $1 \alpha$ regulates other neuronal proteins including synaptotagmin 2 and complexin 1. Although the transcriptional changes in these three genes are similar, we find that the effects on inhibitory synaptic function are opposite between cortex and hippocampus in PGC-1 $\alpha-/-$ mice. Loss of PGC-1 $\alpha$ causes a deficiency in inhibitory synaptic transmission in motor cortex, including reduced GABA release at gamma frequency stimulation. In contrast, mice with deletion of PGC- $1 \alpha$ have enhanced inhibition in hippocampus, leading to reduced circuit activation and increased power of hippocampal gamma oscillations. In both cases, the alterations in inhibition cause behavioral impairment. PGC- $1 \alpha-/-$ mice have severe motor deficits and a deficit in prepulse inhibition, as well as impairment in nest building, a hippocampal-dependent innate behavioral task. Together, our results show that PGC-1 $\alpha$ differentially regulates inhibitory synaptic transmission depending on brain region, and that both increases and decreases in inhibition caused by loss of PGC-1 $\alpha$ are detrimental to normal circuit function. The regional specificity of inhibitory dysfunction caused by alterations in PGC-1 $\alpha$ could be a mechanism contributing to diverse symptoms in a variety of neurological disorders.
\end{abstract}

\title{
Effects of Ketamine and Acetyl 1-carnitine (ALCAR) on the Zebrafish Nervous System
}

\author{
Jyotshna Kanungo \\ Division of Neurotoxicology, National Center for Toxicological Research, US Food and Drug Administration, Jefferson, AR, USA
}

\begin{abstract}
Ketamine, an antagonist of the $\mathrm{N}$-methyl-d-aspartate (NMDA) receptors, is a pediatric anesthetic. As in mammals, ketamine is neurotoxic in zebrafish embryos and ALCAR relieves ketamine's neurotoxic effects. On the other hand, low doses of ketamine act as fast antidepressants. The exact mechanism(s) of how ketamine causes neurotoxicity remains unclear. For mechanistic studies, zebrafish embryos are better suited than higher order vertebrates. Image-based high content analysis and use of transgenic zebrafish embryos show that ketamine induces neuro-developmental toxicity. The sensory and motor neurons as well as the serotonergic and dopaminergic systems are modulated by ketamine but not in the presence of ALCAR indicating beneficial effects of ALCAR on ketamine-induced neurodevelopmental defects. Interference with various pathways in vivo that are potentially involved in inducing ketamine toxicity as well as in vitro data supporting the former will be presented to demonstrate how ALCAR abrogates ketamine's adverse effects on the zebrafish embryos.
\end{abstract}

\section{A Tale of a Tail: The Amyloid-precursor Protein Intracellular Domain is an Effector Molecule of Metaplasticity}

\author{
Jannic Boehm* and Emilie-Trillaud-Doppia
}

Université de Montréal, Canada

\begin{abstract}
Alzheimer disease (AD) is initially characterized as a disease of the synapse that affects synaptic transmission and synaptic plasticity. While amyloid-beta and tau have been traditionally implicated in causing $\mathrm{AD}$, recent studies suggest that other factors, such as the intracellular domain of the amyloid-precursor protein (APP-ICD), can also play a role in the development of AD. We find that the expression of APP-ICD induces synaptic depression, while the intracellular domain of its homolog amyloid-like precursor protein 2 does not. To further analyze how APP-ICD affects synaptic plasticity and to separate these effects from amyloid-beta dependent effects, we created a chimeric APP where the amyloid-beta domain is exchanged for its homologous domain from the amyloid-like precursor protein 2. We show that the expression of this chimeric APP has no effect on basal synaptic transmission or synaptic plasticity. However, a synaptic priming protocol, which in control cells has no effect on synaptic plasticity, leads to a complete block of subsequent induction of long-term potentiation and a facilitation of long-term depression in neurons expressing chimeric APP. Our results therefore indicate that the APP intracellular domain affects metaplasticity, i.e. it changes the ability of synapses to induce synaptic plasticity. We show that the underlying mechanism for changes in metaplasticity is due to caspase cleavage of the APP-ICD and involves activation of post-synaptic ryanodine receptors. Taken together, our results provide new insights into the altered regulation of synaptic plasticity during $\mathrm{AD}$.
\end{abstract}




\title{
Test-retest Reliability of Graph Metrics of Resting State MRI Functional Brain Networks: A Review
}

\author{
Martina Andellini ${ }^{1^{*}}$, Vittorio Cannata ${ }^{1}$, Simone Gazzellini $^{2}$, Bruno Bernardi ${ }^{3}$ and Antonio Napolitano ${ }^{1}$ \\ ${ }^{1}$ Medical Physics Department, Enterprise Risk Management, Bambino Gesù Children's Hospital, Rome, Lazio, Italy \\ ${ }^{2}$ Department of Neuroscience and Neurorehabilitation, Bambino Gesù Children's Hospital, Rome, Lazio, Italy \\ ${ }^{3}$ Unit of Neuroradiology, Bambino Gesù Children's Hospital, Rome, Lazio, Italy
}

\begin{abstract}
The employment of graph theory to analyze spontaneous fluctuations in resting state BOLD fMRI data has become a dominant theme in brain imaging studies and neuroscience. Analysis of resting state functional brain networks based on graph theory has proven to be a powerful tool to quantitatively characterize functional architecture of the brain and it has provided a new platform to explore the overall structure of local and global functional connectivity in the brain. Due to its increased use and possible expansion to clinical use, it is essential that the reliability of such technique be very strongly assessed. In this review, we explore the outcome of recent studies in network reliability, which apply graph theory to analyze connectome resting state networks. Therefore, we investigate which preprocessing steps may affect reproducibility the most. In order to investigate network reliability, we compared the test-retest (TRT) reliability of functional data of published neuroimaging studies with different preprocessing steps. These data were combined in a meta-summary reliability analysis to investigate, on a larger population, the effects of preprocessing and processing steps on the reliability of functional connectivity. In particular, we tested influence of global signal regression, correlation metric choice, binary versus weighted link definition, frequency band selection and length of time-series, combining in a common analysis ICC values from multiple studies. Statistical analysis shows that only frequency band selection and length of time-series seem to affect TRT reliability. Our results highlight the importance of the choice of the preprocessing steps to achieve more reproducible measurements.
\end{abstract}

\section{Analysis of Volatile Organic Compounds (VOCs): A Possible Application for Early Detection of Parkinson's Disease}

Soliman Khatib ${ }^{1,2^{*}}$, John Finberg ${ }^{3}$, Fadi Artoul ${ }^{1}$, Yizhar Lavner ${ }^{2}$, Saeed Mahmood ${ }^{1}$, Ulrike Tisch ${ }^{3}$, Hossam Haick ${ }^{3}$, Yuval Aluf ${ }^{1,3}$ and Jacob Vaya ${ }^{1,2}$

${ }^{1}$ MIGAL-Galilee Research Institute, Israel

${ }^{2}$ Tel-Hai College, Israel

${ }^{3}$ Technion-Institute of Technology, Israel

\begin{abstract}
Parkinson's disease (PD) is characterized by dopaminergic (DA) neuron depletion. Early detection of PD may help in selecting the appropriate treatment. Biomarkers of PD have been suggested, however none of these is currently in clinical use. The aim of the study is to identify volatile organic compounds (VOCs) as early biomarkers of PD. Our hypothesis is that during PD progression, specific VOCs are generated that are linked to the biochemical pathways characterizing PD. These VOCs can be detected by GC-MS combined with solid-phase microextraction (SPME) technique. Three groups of rats were studied: DAlesioned rats injected with 6-hydroxydopamine (HDA; $250 \mu \mathrm{g} / \mathrm{rat} \mathrm{n}=11$ ); control rats injected with saline $(\mathrm{n}=9)$, and control rats injected with DSP-4 ( $\mathrm{n}=8)$, a specific noradrenergic neuron toxin. Blood and striatal tissue homogenate were analyzed. In the blood, 1-octen-3-ol and 2-ethylhexanol were found at significantly higher concentrations in HDA versus sham rats. In the striatal homogenate 1-octen-3-ol and other four compounds were found at significantly lower concentrations in HDA versus sham rats. 1-Octen-3-ol is a cytotoxic compound. These results may lead to the development of an early diagnostic test for PD based on profiling of VOCs in body fluids.
\end{abstract}

\section{Predictive Value of Human Skin Fibroblast Biomarkers for Alzheimer's Disease using a Biomarker Severity Score}

Florin V. Chirila* and Daniel. L. Alkon

Blanchette Rockefeller Neurosciences Institute, Morgantown, WV, USA 


\begin{abstract}
The value of diagnostic biomarkers derives from their ability to monitor disease progression and remission, as well as their predictive accuracy before the onset of the disease. Detection of the disease even before its onset, could provide important opportunities for prevention and/or planning therapeutic strategies. In the early stages of Alzheimer's disease (AD), within four years from the dementia onset, clinical diagnosis has a limited rate of success. Furthermore, clinical diagnostic accuracy before dementia onset has not been previously validated. Here, we present evidence for the potential predictive value of three peripheral human biomarkers for Alzheimer's disease (AD): Morphometric Imaging, PKC $\varepsilon$ and AD Index. The Biomarker Severity Score as described herein may be a continuous logistic fit function on the normalized values, between 0 and 100\%, of the output signal of the biomarkers for the Age-matched control (AC) and Alzheimer's disease (AD) patients. The gap in the severity score between the $\mathrm{AD}$ patients and $\mathrm{AC}$ patients is greater than $40 \%$ for each biomarker and indicates that each of these three biomarkers have the potential to detect the signature of Alzheimer's disease several years before the dementia onset. Strategies for detecting $\mathrm{AD}$ before dementia onset with these biomarkers are presented here.
\end{abstract}

\title{
Diagnosis and Treatment of Late-onset Pompe Disease in the Middle East and North Africa Region: Consensus Recommendations from an Expert Group
}

\author{
A1 Jasmi F ${ }^{1}$, Al Jumah $\mathrm{M}^{2,3}$, Alqarni F ${ }^{4}$, Al-Sanna'a N ${ }^{5}$, Al-Sharif F $\mathrm{F}^{6}$, Bohlega $\mathrm{S}^{7}$, Cupler EJ ${ }^{8}$, Fathalla $\mathrm{W}^{9}$, Hamdan MA ${ }^{10}$, \\ Makhseed $\mathrm{N}^{11}$, Nafissi $\mathrm{S}^{12}$, Nilipour $\mathrm{Y}^{13}$, Selim L ${ }^{14}$, Shembesh $\mathrm{N}^{15}$, Sunbul $\mathrm{R}^{16}$ and Tonekaboni $\mathrm{SH}^{17}$ \\ ${ }^{1}$ Department of Pediatrics, College of Medicine and Health Science, United Arab Emirates University, Al-Ain, United Arab Emirates \\ ${ }^{2}$ King Abdullah International Medical Research Center, King Saud Bin Abdulaziz University for Health Sciences, NGHA, Riyadh, \\ Kingdom of Saudi Arabia \\ ${ }^{3}$ Prince Mohammed Ben Abdulaziz Hospital, Riyadh, Kingdom of Saudi Arabia \\ ${ }^{4}$ Neurology Department, National Neurosciences Institute, King Fahad Medical City, Riyadh, Kingdom of Saudi Arabia \\ ${ }_{5}^{5}$ Johns Hopkins Aramco Healthcare, Pediatrics Services Division, Dhahran, Kingdom of Saudi Arabia \\ ${ }^{6}$ Medical Genetics and Metabolic Consultant, Jeddah, Kingdom of Saudi Arabia \\ ${ }^{7}$ Department of Neurosciences, MBC 76, King Faisal Specialist Hospital and Research Centre, Riyadh, Kingdom of Saudi Arabia \\ ${ }^{8}$ Department of Neuroscience, MBCJ-76, King Faisal Specialist Hospital and Research Center, Jeddah, Kingdom of Saudi Arabia \\ ${ }^{9}$ Department of Pediatrics, Division of Child Neurology, Mafraq Hospital, Abu Dhabi, United Arab Emirates \\ ${ }^{10}$ KidsHeart: American Fetal \& Children's Heart Center, Dubai Healthcare City, Dubai, United Arab Emirates \\ ${ }^{11}$ Pediatric Department, Jahra Hospital, Ministry of Health, Qadisiya, Kuwait \\ ${ }^{12}$ Department of Neurology, Tehran University of Medical Sciences, Shariati Hospital, Tehran, Iran \\ ${ }^{13}$ Pediatric Pathology Research Center, Mofid Children Hospital, Shahid Beheshti Medical University (SBMU), Shariati Avenue, \\ Tehran, Iran \\ ${ }^{14}$ Pediatric Neurology and Neurometabolic Division, Cairo University Children Hospital (Abo el Reesh), Cairo, Egypt \\ ${ }^{15}$ Pediatrics and Pediatric Neurology, Benghazi University, Benghazi, Libya \\ ${ }^{16}$ Department of Pediatrics, Qatif Central Hospital, Dammam, Eastern Province, Kingdom of Saudi Arabia \\ ${ }^{17}$ Pediatric Neurology Research Center, Mofid Children Hospital, Shahid Beheshti Medical University (SBMU), Shariati Avenue, \\ Tehran, Iran
}

\begin{abstract}
Pompe disease is a rare autosomal recessive disorder caused by a deficiency of the lysosomal enzyme alpha-glucosidase. Late-onset Pompe disease has a complex multisystem phenotype characterized by a range of symptoms which has led to underestimate the disease among adult population. Consensus-based guidelines for the diagnosis and treatment of late-onset Pompe disease was created by an expert panel from the Middle East and North Africa (MENA) region, where the relative prevalence of Pompe disease is thought to be high but there is a lack of awareness and diagnostic facilities. These guidelines set out practical recommendations and include algorithms for the diagnosis and treatment of late-onset Pompe disease. They detail the ideal diagnostic workup, indicate the patients in whom enzyme replacement therapy should be initiated, and provide guidance on appropriate patient monitoring. These guidelines will serve to increase awareness of the condition, optimize patient diagnosis and treatment, reduce disease burden, and improve patient outcomes. In addition, we recently diagnosed the first two patients with Late-onset Pompe disease in the middle east.
\end{abstract}




\title{
The Role of Clinical Proteomics, Lipidomics and Genomics in the Diagnosis of Alzheimer's Disease
}

\author{
Ian James Martins
}

School of Medical Sciences/Edith Corwan University, Australia

\begin{abstract}
The early diagnosis of Alzheimer's disease $(\mathrm{AD})$ has become important to the reversal and treatment of neurodegeneration that may be relevant to premature brain aging that is associated with chronic disease progression. Clinical proteomics allows the detection of various proteins in fluids such as the urine, plasma and cerebrospinal fluid for the diagnosis of AD. Interests in lipidomics has accelerated with plasma testing for various lipid biomarkers that may with clinical proteomics provide a more reproducible diagnosis. The combination of proteomics with lipidomics may decrease the biological variability between studies and provide reproducible results that detect a community's susceptibility to AD. The diagnosis of chronic disease associated with AD that now involves genomics may provide increased sensitivity to avoid inadvertent errors related to plasma versus cerebrospinal fluid testing by proteomics and lipidomics that identify new disease biomarkers in body fluids, cells and tissues. The diagnosis of $\mathrm{AD}$ by various plasma biomarkers with clinical proteomics may now require the involvement of lipidomics and genomics to provide interpretation of proteomic results from various laboratories around the world. Technological advances allow novel biomarkers to be identified for the diagnosis of neurodisorders but novel patents may not be relevant to diagnostic discovery with relevance to autonomous disease that involves magnesium and its association with various chronic diseases.
\end{abstract}

\section{The Neuroprotective Effects of Physical Activity for Persons at Risk for Alzheimer's Disease}

\section{Cristy Phillips}

Arkansas State University, AR, USA

\begin{abstract}
Convergent evidence suggests that regular physical activity $(\mathrm{PA})$ optimizes the function of neural mechanisms underlying cognition in aging persons at risk for Alzheimer's disease (AD). Preclinical and clinical studies demonstrate that PA optimizes synaptic number and function, neurogenesis, neurotrophin levels, immune function, and circadian rhythm while simultaneously ameliorating processes involved in plaque and neurofibrillary tangle formation. Notwithstanding, more than one-third of the world adult population fails to meet recommended activity guidelines. Given the extended prodromal phase of AD, ample time exists to harness these health-related benefits. In this session, I will discuss the protocols that have been used to alter the progression of AD-related neuropathology and highlight implications for practice.
\end{abstract}

\section{Effects of Problem Solving Therapy on Depression and Anxiety Levels in Family Caregivers of Persons with a New Diagnosis of Mild Cognitive Impairment or Early Dementia: A Randomized Controlled Trial}

\author{
Linda Garand ${ }^{1 *}$, Mary Amanda Dew ${ }^{2}$, Charles F. Reynolds III ${ }^{3}$ and Oscar L. Lopez ${ }^{4}$ \\ ${ }^{1}$ Duquesne University School of Nursing, PA, USA \\ ${ }^{2}$ University of Pittsburgh Schools of Medicine and Public Health, PA, USA \\ ${ }^{3}$ University of Pittsburgh School of Medicine, PA, USA \\ ${ }^{4}$ Director, Alzheimer Disease Research Center, PA, USA
}

\begin{abstract}
Objective: Interventions directed at the mental health of family dementia caregivers may have limited impact when focused on caregivers who have provided care for years and report high burden levels. We sought to evaluate the mental health effects of problem-solving therapy (PST), designed for caregivers of individuals with a recent diagnosis of Mild Cognitive Impairment (MCI) or early dementia.
\end{abstract}

Method: Seventy-three (43 MCI and 30 early dementia) family caregivers were randomly assigned to receive PST or a 
comparison condition (nutritional education). Depression, anxiety, and problem-solving orientation were assessed at baseline and at $1,3,6$, and 12 months post intervention.

Results: PST had beneficial effects on caregivers' depression and anxiety levels, with the positive mental health outcomes being more pronounced in the early dementia (versus MCI) caregivers. Symptoms of depression were reduced in early dementia caregivers and prevented from rising in MCI caregivers when PST was taught soon (within six months) after receiving the cognitive diagnosis. While PST reduced anxiety levels in the early dementia caregivers, anxiety levels of MCI caregivers remained relatively low over time, regardless of study group assignment. We also found that the PST intervention had a significant impact on the caregiver's "problem orientation" or attitude that problems are solvable.

Discussion: Enhanced problem-solving skills, learned early after a loved one's cognitive diagnosis (especially dementia), results in positive mental health outcomes among new family caregivers.

\title{
Palmitate-induced ER Stress and Subsequent CHOP Activation Attenuates Leptin and IGF1 Expression - Implications for Alzheimer's Disease
}

\author{
Othman Ghribi* and Gurdeep Marwarha \\ Department of Biomedical Sciences, University of North Dakota School of Medicine, ND, USA
}

\begin{abstract}
Several studies suggest the therapeutic potential of the adipocytokine leptin and the growth factor, Insulin-like Growth Factor (IGF1), in delaying the progression of Alzheimer's disease (AD). We have previously shown that the cholesterol oxidized product 27-hydroxycholesterol inhibits leptin expression, by evoking endoplasmic reticulum (ER) stress, a cellular response that maybe implicated in AD. Several studies have posited a positive correlation between saturated fat intake and the risk of developing AD. Palmitic acid (Palmitate) is the most abundant long-chain saturated fatty acid (sFFA) in the brain. However, the extent to which palmitate induces ER stress and consequently attenuates leptin and IGF1 expression and contributes to generating AD-like pathology has not been determined. In this study, we demonstrate inSH-SY5Y human and Neuro2a mouse neuroblastoma cells, as well as the mouse brain that palmitate attenuates leptin and IGF1 expression by inducing $\mathrm{ER}$ stress and activating C/EBP Homologous Protein (CHOP), which negatively regulates $\mathrm{C} / \mathrm{EBP} \alpha$, a transcription factor required for leptin and IGF1 expression. Pretreatment with 4-phenylbutyric acid, a molecular chaperone that alleviates ER stress, precluded the palmitate-evoked down-regulation of leptin and IGF1. Furthermore, we demonstrate that the activation of the transcription factor CHOP in response to palmitate-induced ER stress is pivotal in the mitigation of leptin and IGF1 expression as knocking-down CHOP alleviates the palmitate-induced abrogation in leptin and IGF1 expression. Our study implicates ER stress as the mechanistic link in the palmitate-induced negative regulation of leptin and IGF1, two cytokines that have potential therapeutic effects in $\mathrm{AD}$.
\end{abstract}

\section{Protein Aggregation as a Link Between Diabetes and Alzheimer's Disease}

\author{
Gunilla T. Westermark ${ }^{*}$, Martin Ingelsson ${ }^{2}$, Isabel Costantino ${ }^{3}$, Christopher William ${ }^{3}$, Marta Marquie-Sayagues ${ }^{3}$, \\ Matthew P. Frosch ${ }^{3}$, Bradley T. Hyman ${ }^{3}$ and Marie E. Oskarsson ${ }^{1}$ \\ ${ }^{1}$ Medical Cell Biology, Uppsala University, Sweden \\ ${ }^{2}$ Department of Public Health/Geriatrics, Uppsala University, Sweden \\ ${ }^{3}$ Department of Neurology/Alzheimer's Disease Research Unit, Massachusetts General Hospital, Harvard Medical School, Boston, MA, USA
}

\begin{abstract}
Alzheimer disease (AD) and type 2 diabetes (T2D) are two age-related diseases that share the formation of local deposits of amyloid. In $\mathrm{AD}, \mathrm{A} \beta$ constitutes the main protein component of the brain deposits and cerebral amyloid angiopathy (CAA) while islet amyloid polypeptide (IAPP) forms local amyloid in the islets of Langerhans in patients with T2D. T2D has been identified as a risk factor for AD. We have shown that preformed fibrils made from $\mathrm{A} \beta$ can function as an in vivo seed and propagate IAPP amyloid in the islets of Langerhans in human IAPP transgenic mice. Also, with the use of the sensitive and highly specific PLA-technique we have shown that IAPP and A $\beta$ co-localize in plaques and vessel wall amyloid in AD patients. Presently, we have compared the plaque profile of patients with $\mathrm{AD}+\mathrm{T} 2 \mathrm{D}$ with the profile of patients with only $\mathrm{AD}$. Congo red staining and immunohistochemistry with $A \beta$-antibody on cortical sections revealed a higher percentage of neuritic plaques in $\mathrm{AD}+\mathrm{T} 2 \mathrm{D}$. No difference in degree of co-localization of $\mathrm{A} \beta$ and IAPP in plaques using PLA was observed between AD+T2D and $\mathrm{AD}$ groups. Western blot analysis of tissue extracts showed a reduced IAPP concentration in the AD+T2D group. The
\end{abstract}


result points to a continuous addition of IAPP to the A $\beta$ plaques. The lower IAPP load in AD+T2D is interpreted as an effect of reduced $\beta$-cell mass or function. Detected differences in end-stage pathology observed between $\mathrm{AD}$ patients with and without T2D indicate differences in pathogenesis. Further studies are needed.

\title{
Implication of Protein Metabolism in the Pathogenesis of Alzheimer's Disease
}

\author{
Yves Ingenbleek \\ University Louis Pasteur, Laboratory of Nutrition, Strasbourg, France
}

\begin{abstract}
Human body composition studies indicate that nitrogen $(\mathrm{N})$ is an essential component of all proteins found in body tissues. The bulk of $\mathrm{N}$ stores is confined within the lean body mass (LBM) whose evolutionary patterns from birth to old age are closely superimposable to those of transthyretin (TTR), hence promoting this analyte as the best surrogate biomarker of LBM fluctuations. Protein malnutrition leads to unachieved LBM replenishment, defective release of reductant molecules involved in the synthesis of hydrogen sulfide (H2S) and overproduction of homocysteine (Hcy) required for the maintenance of methionine homeostasis. Inflammatory disorders generate cytokine-induced increased $\mathrm{N}$ urinary losses, leading similarly to LBM downsizing and subnormal TTR values in plasma and cerebrospinal fluid. In that context, TTR loses its normal neuroprotective properties and its capacity to dismantle $A \beta$ amyloid products in cerebral tissues, as long as malnutrition/ inflammation burden persists. Worsening process of Alzheimer's disease might result from growth of reactive oxygen species (ROS) in brain tissues, arising from the combined effect of impaired H2S production and high Hcy values.
\end{abstract}

\section{Anti-dipeptidyl-peptidase-like Protein-6 Antibody Dementia}

\section{Gorazd Bernard Stokin}

International Clinical Research Centre, St. Anne's University Hospital, Brno, Czech Republic Division of Neurology, University Medical Centre, Ljubljana, Slovenia

\begin{abstract}
Recent studies uncovered a series of autoimmune encephalitides manifesting largely with behavioral changes and cognitive impairment that often murk the boundary between rapidly and more common slowly progressive dementias. One such example is the Anti-Dipeptidyl-Peptidase-Like Protein-6 antibody encephalitis. Anti-Dipeptidyl-Peptidase-Like Protein-6 antibody encephalitis is characterized by gastrointestinal symptoms followed by behavioral changes and cognitive impairment in addition to a plethora of other for now less consistent neurological symptoms and signs. Pathologically, it consists in neurodegeneration coupled to inflammatory infiltration by CD8 positive T-lymphocytes. This contribution reports a clinical-pathological case of a patient with Anti-Dipeptidyl-Peptidase-Like Protein-6 antibody encephalitis and reviews the current knowledge of this novel autoimmune encephalitis.
\end{abstract}

\section{The Role of Arterial Stiffness in Assessment of Cognitive Decline}

\author{
Sandra Morovic \\ Department of Neurology, Aviva Medical Center, Croatia
}

\begin{abstract}
Cognitive impairment is still considered as a normal side effect of aging in general population. Greater population life expectancy leads to increased incidence of cognitive impairment cases, making this one of the most important medical and social problems worldwide. Therefore, prevention of cognitive impairment is an imperative. Dementia includes a heterogeneous group of disorders, the most common being Alzheimer dementia (AD) and vascular dementia (VD). Most cardiovascular risk factors, such as hypertension, diabetes mellitus, hypercholsterolemia, atrial fibrillation, and smoking are not exclusively risk factors for $\mathrm{VD}$, but also for AD. Early changes of the blood vessel wall can be detected by early ultrasound screening methods which allow us to detect changes before the disease becomes clinically evident. Intracranial hemodynamics can be assessed by Transcranial Doppler Sonography (TCD), functional TCD with various functional tests, and TCD detection of cerebral emboli. Extracranial circulation, carotid and vertebral arteries, can be assessed by means of color Doppler flow imaging (CDFI). Novel ultrasound
\end{abstract}


technology enables us non-invasive, bedside detection of early vascular changes such as arterial stiffness, measurement of the intima-media thickness (IMT), pulse-wave velocity, or endothelial dysfunction in order to obtain information necessary to closer determine the relation between vascular status and disease development, so that the evolution of CVD could be prevented or at least postponed. Early disease detection enables on-time management of vascular risk factors, and can postpone or even reverse disease progression.

\title{
Targeting'Therapy for Homocysteic Acid in Blood is an Alzheimer's Cognitive Recovery
}

\author{
Tohru Hasegawa ${ }^{*}$ and Wataru Ukai \\ ${ }^{1}$ Saga Woman Junior College, Saga, Japan \\ ${ }^{2}$ Sapporo Medical University, Sapporo, Japan
}

\begin{abstract}
At present we have no hope to recover the Alzheimer cognitive impairment. Just only an intervention which combines an exercise with DHA supplement established to recover the cognition. It is interested in DHA supplement combination. Many hypotheses are considered why these interventions can recover the cognitive decline in AD. The exercise indeed stimulates the blood circulation which induces the urinary excretion of blood unknown pathogens for the cognitive impairment and consequently some recovery can be observed. We hypothesize that the homocysteic acid in blood is the one of pathogens which are excreted into urine. Why is DHA supplement needed for the recovery process induced by an exercise? DHA is known to decrease homocysteine level and exercise contributes DHA effect. In other words, the combination of exercise and DHA induces the strong decrease of homocysteine in blood, which supports our hypothesis, the targeting homocysteic acid in blood is a possible method which can recover an Alzheimer cognitive impairment. Our hypothesis was proved by the fact that the memorial problem of $3 \mathrm{xTg}-\mathrm{AD}$ model mice which were developed by amyloid pathology and the model for the familial AD were recovered by anti-HA antibody and not by amyloid treatment. Why did 3xTg-AD mice which increased amyloid pathology increase HA level? APP and/or presenilin increased calcium influx which could increase superoxide level and consequently increase HA level from homocysteine or methionine. Also our hypothesis is partially supported by the open clinical trial of some supplement which can decrease the homocysteic acid in blood for Alzheimer's patients and the result is very impressive. Our hypothesis, the destruction of HA will induce the cognitive reserve ability and its realization is urgent hypothesis to be solved.
\end{abstract}

\section{Prefibrillar Tau Oligomers Alter the Nucleic Acid Protective Function of Tau in Hippocampal Neurons in vivo}

\author{
Marie-C Galas ${ }^{*}$, Marie Violet ${ }^{1}$, Alban Chauderlier ${ }^{1}$, Lucie Delattre ${ }^{1}$, Meryem Tardivel ${ }^{1}$, Lester Binder ${ }^{2}$, Rakez Kayed ${ }^{3,5}$, \\ Bruno Lefebvre ${ }^{1}$, Eliette Bonnefoy ${ }^{4}$ and Luc Buee ${ }^{1}$ \\ ${ }^{1}$ INSERM UMRS1172, JPArc, Alzheimer E Tauopathies, France \\ ${ }^{2}$ Department of Translational Science $\Xi^{\circ}$ Molecular Medicine, College of Human Medicine, Michigan State University, MI, USA \\ ${ }^{3}$ Department of Neurology, George and Cynthia Mitchell Center for Neurodegenerative Diseases, University of Texas Medical Branch, \\ $T X, U S A$ \\ ${ }^{4}$ INSERM UMRS1007, Universite'Paris Descartes, France \\ ${ }^{5}$ Department of Neuroscience $\Xi^{\circ}$ Cell Biology, George and Cynthia Mitchell Center for Neurodegenerative Diseases, University of Texas \\ Medical Branch, TX, USA
}

\begin{abstract}
In addition to its well-known role in microtubule stabilization, Tau is a key player in the protection of nuclear DNA integrity in neurons under physiological and ROS---producing stress conditions. Surprisingly, in vivo, Tau deficiency also altered the integrity of cytoplasmic and nuclear RNA, suggesting a more general role of Tau in nucleic acid protection. We tested the impact of Tau pathological forms on nucleic acid integrity in vivo, in a mouse model of tauopathy (THY---Tau22). Our results demonstrated that hyperthermia (Hyp)---induced nucleic acid damage is strictly correlated with prefibrillar Tau oligomers formation. A similar association between prefibrillar Tau oligomers and nucleic acid oxidative damage was observed in AD brains. Pretreatment with Methylene Blue, a Tau aggregation inhibitor and a redox cycler, reduced Hyp---induced Tau oligomerization as well as nucleic acid damage. This study clearly highlights the existence of an early and critical time frame for Hyp---induced Tau oligomerization, which most likely occurs through increased oxidative stress, and nucleic acid vulnerability during the progression of Tau pathology. These results suggest that at early stages of AD, Tau oligomerization triggers the loss
\end{abstract}


of the nucleic acid protective function of monomeric Tau. It highlights the existence of a short therapeutic window in which to prevent the formation of pathological forms of Tau and their harmful consequences on nucleic acid integrity during the progression of Tau pathology.

\title{
Early Non-Invasive Treatment of Non-Salient Accelerated Cognitive Decline in Late- life Ageing Humans
}

\section{Oded Meiron}

Herzog Hospital, Clinical Research Center for Brain Sciences, Jerusalem, Israel

\begin{abstract}
Accelerated decline in verbal memory and everyday functioning in old age has been linked to neuropathological processes in the prefrontal cortex, reduced medial temporal lobe volume, and decreased hippocampal activation, particularly in patients suffering from various forms of dementia. Pharmacological treatments have failed to delay the onset, or reduce the severity of, debilitating late-life neurodegenerative cognitive impairments. In line with our recent findings that indicated enhanced verbal working memory performance and episodic memory improvement following bi-frontal theta-rhythm transcranial current stimulation (tACS) in healthy humans, we propose a plausible therapeutic-intervention paradigm directed to enhance executive working memory maintenance and memory consolidation in older individuals (ages 50-80) diagnosed with mild cognitive impairment (MCI) or with mild-to-moderate dementia (i.e., first stages after Alzheimer's disease onset). Resting electroencephalography (EEG), sensory EEG event-related potentials (ERP), and executive attention deficits are estimated to differentiate accelerated cognitive impairment symptoms versus normal ageing cognitive deficits. Further electrophysiological and behavioral data collection in older adults (60-80 years old) could lead to the development targeted non-invasive personalized transcranial current stimulation treatments to enhance and preserve cortical network-synchrony and executive cognitive functions in non-demented ageing individuals and in clinically-high-risk prodromal dementia patients. Therefore, we suggest a preliminary path towards developing a behaviorally-based electrophysiological marker to denote and guide the early noninvasive treatment of MCI symptoms in late-life ageing humans.
\end{abstract}

\section{Self-Administered Gerocognitive Examination (SAGE) Correlates to Montreal Cognitive Assessment (MOCA) in a Suburban Neurology Clinic}

\author{
Ansh Bhammar*, Heena Patel, Malini Nair and Anil Nair \\ Alzheimer Disease Center, Boston, MA, USA
}

\begin{abstract}
Background: Self-Administered Gerocognitive Examination (SAGE) and Montreal Cognitive Assessment (MOCA) are commonly used evaluation tools in geriatrics. SAGE is a relatively new geriatric neurocognitive exam while MOCA is generally considered an established marker for cognitive assessment in the clinical setting. We compared the scores on SAGE to MOCA scores corrected for education among patients attending the www.alzcenter.com clinic.
\end{abstract}

Method: We collected SAGE scores, education-corrected MOCA scores, education, age, gender and race from patients attending a suburban neurology clinic from 2010-2016. As data were not normally distributed, spearman correlation tests were used. Linear models were used for multivariate analyses.

Results: SAGE scores were significantly correlated to corrected MOCA scores (rho = 0.63, $\mathrm{p}<0.0001$ ). After adjusting for age, gender and race effects, the association persisted $(\mathrm{p}<0.0001)$.

Conclusion: SAGE scores correlated to education-corrected MOCA scores among patients attending a suburban neurology clinic. 


\title{
Post-traumatic Stress Disorder Symptoms in Patients with Pituitary Adenomas before Surgery and at Three Months After Tumor Removal
}

\author{
Florian Bruckbauer ${ }^{13^{*}}$, Youssef Shiban ${ }^{2}$, Frank H. Wilhelm ${ }^{2}$, Jens Lehmberg ${ }^{1}$, Bernhard Meyer $^{1}$ and Ehab Shiban $^{1}$ \\ ${ }^{1}$ Department of Neurosurgery, Technical University Munich, Germany \\ ${ }^{2}$ Department of Experimental Psychology (Clinical Psychology and Psychotherapy), University of Regensburg, Germany \\ ${ }^{3}$ Department of Psychology, University of Salzburg, Austria
}

\begin{abstract}
Objective: Patients with pituitary tumors express symptoms of psychological disruption and impaired quality of life. Alterations in the various hormonal axes affected by the tumor with connections from the hypothalamus to other limbic structures may cause depression, anxiety and mood disorders. Aim of this study was to compare clinically relevant scores for post - traumatic stress, depression and anxiety in patients before and after brain tumor removal.
\end{abstract}

Methods: An exploratory analysis of prospective data of patients undergoing endonasal tumor resection was performed. The patients were evaluated for posttraumatic stress (PTSS score), depression (CES-D score) and anxiety (STAI-S/T, ASI-3) and health related quality of life $(\mathrm{SF}-36)$ before surgery and at three months followup.

Results: Eight patients (5 male, 3 female) met all inclusion criteria, mean age was 63 years. Abnormal PTSS scores were reported in $63 \%$ of cases preoperatively and in $25 \%$ of cases three months after surgery. Before surgery, abnormal STAI-S, STAI-T and ASI-3 scores were encountered in 75\%, 50\% and 25\% of patients, respectively, and postoperatively in $63 \%, 38 \%$ and $13 \%$ of patients. Abnormal CES-D scores before surgery were detected in $38 \%$ of patients and in $13 \%$ at three months follow-up.

Discussion: Descriptively, three months after tumor removal fewer patients with pituitary adenomas show abnormal scores for post-traumatic stress, anxiety and depression. The hypothesized link between tumor resection und symptom reduction will be evaluated in a larger, controlled follow-up study.

\section{Age-related Differences in Executive Functions}

\author{
Dorota Buczylowska and Franz Petermann \\ Center for Clinical Psychology and Rehabilitation, University of Bremen, Germany
}

\begin{abstract}
Executive functions (EFs) are the higher mental processes responsible for the coordination of cognitive operations. They are some of the earliest cognitive functions to deteriorate with normal aging. Age-related interindividual variability in EFs is not well studied. However, this issue should be investigated as studying the differences between individuals may help explain why some individuals change in EFs over time, whereas others do not.
\end{abstract}

Method: We used data of 484 participants aged 18-99 years from the German adaptation of the Neuropsychological Assessment Battery (NAB) to examine age-related differences in EFs.

Results: Analyses showed an increase in the variability of scores with age, varying from $7 \%$ to $289 \%$, in all NAB Executive Functions Module subtests. Moreover, age-dependent variability seemed to be associated with age-dependent decline because the subtests with the greatest increase in dispersion, (i.e., Mazes, Planning, and Categories), also showed the greatest decrease in mean scores. In contrast, scores for the subtests Letter Fluency, Word Generation, and Judgment showed the lowest increase in dispersion with the lowest decrease in mean scores.

Conclusions: The results showed a pattern of age-related differences in executive functioning that is consistent with the concept of crystallized and fluid intelligence. It may be concluded that EF tasks with a pronounced crystallized component are less sensitive to age-dependent deterioration than those linked to fluid intelligence. These findings might be useful in the assessment of mild, subclinical executive dysfunction in normal adults and help enhance the neuropsychological diagnostics in patients with brain lesions. 


\title{
A Brainstem Hypothesis for Autism
}

\author{
Woody R. McGinnis \\ Autism Research Institute, San Diego, CA, USA
}

\begin{abstract}
We explore the hypothetical relationship of impaired function of nucleus tractus solitarius (NTS) to the behavioral and visceral findings in autism. NTS is brain's primary point-of-entry for visceral afference, and experimental interruption of visceral afference replicates cardiovascular, gastrointestinal, immunological, and laryngeal abnormalities reported in autism. Neurovisceral afference is known to sub serve higher functions such as mood and memory, and impairment of NTS also is predicted to exert direct, widespread effects on perfusion and inflammation of higher regions of brain in autism. Unusual microvascular anatomy is proposed to render NTS preferentially sensitive to both hypoxic and toxic injury, and in autism these two insults may intertwine. The epidemiologic data strongly associate autism with hypoxic perinatal events, and also with environmental exposure to neurotoxic cations that are expected to concentrate at NTS. The hypothesis kindles interest in a broad class of cationic neurotoxins (MSG, mercury, cadmium, fluoride) that are usually excluded from consideration as triggers for autistic regression because they do not readily transit the blood-brain barrier.
\end{abstract}

\section{Investigating Interventions and Service Provision for Deaf/Hard of Hearing Students with Autism Spectrum Disorder}

\author{
Stacey Jones Bock*, Christy Borders and Kristi Probst \\ Illinois State University, Normal, IL, USA
}

\begin{abstract}
Students who are Deaf/Hard of Hearing (DHH) with Autism Spectrum Disorder (ASD) are an increasingly discussed population. Much of the discussion however still gravitates around characteristics with few diving into interventions. This may occur due to the overlapping characteristics of hearing loss and ASD (Knoors \& Vervloed, 2011). While there are more and more researchers diving into research on this population, intervention research is still limited at this time. Professionals have suggested initial directions for addressing this population. A review of the literature indicated 11 studies related to interventions used and service provision for students with comorbid D/HH and ASD. There were seven studies that addressed specific interventions (Reinforcement-Easterbrooks \& Handley, 2005; Mace, Pratt, Prager, \& Pritchard, 2011; Video Modeling-Thrasher, 2014; PECS-Malandraki \& Okalidou, 2007; Sign Language-Denmark, Atkinson, Campbell, \& Swettenham, 2014; Shield, 2014) two related to provision of services or practices (Borders, Bock, \& Szymanski, 2015; Guardino, 2015), and two addressing differential diagnosis (Mood \& Shield, 2014; Myck-Wayne, Robinson, \& Henson, 2011). This presentation will discuss the results of this review of literature and suggested modifications to interventions will be discussed. Further, implications for the fields of autism and directions for future research will be discussed.
\end{abstract}

\section{Visual Social Attention in ASD: Reviews and Meta-analyses of Eye-tracking Studies}

\author{
Meia Chita-Tegmark \\ Boston University, MA, USA
}

\begin{abstract}
Determining whether social attention in Autism Spectrum Disorder (ASD) is reduced, whether its distribution is atypical and what factors influence it is important to our theoretical understanding of developmental trajectories of ASD and to designing targeted interventions for ASD. Eye-tracking technology has facilitated research of social attention and results from experimental studies correlate with measures of social impairment and with autism symptom severity. For example, studies have found that reduced attention to social stimuli or increased attention to non-social stimuli is correlated with behavioral measures of autism. (Bird, Press, \& Richardson, 2011; Chawarska, Macari, \& Shic, 2012; Klin, Jones, Schultz, Vokmar \& Cohen, 2002, Shic, Bradshaw, Klin, Scassellati, \& Chawarska, 2011).
\end{abstract}

However, so far no consensus has been reached on whether social attention is fundamentally reduced or absent in individuals with ASD, with some studies showing significantly diminished attention to social information in ASD compared to typically 
developing (TD) controls ( e.g. Klin et al., 2002; Kirchner, Hatri, Heekeren \& Dziobek, 2011; Riby \& Hancock, 2009; Riby, Hancock, Jones, Hanley, 2013; Rice, Moruchi, Jones, Klin, 2012; Shi et al., 2015; Shic, Bradshaw, Klin, Scassellati, \& Chawarska, 2011), while other studies show no differences (e.g. Birmingham, Cerf \& Adolphs, 2011; Freeth, Chapman, Ropar \& Mitchell, 2010; Freeth, Ropar, Mitchell, Chapman, \& Loher, 2011; van der Geest, Kemner, Camfferman, Verbate \& van Engeland, 2002; Kemner, van der Geest, Verbaten, van Engeland, 2007; Kuhn, Kourkoulou, \& Leekam, 2010; Marsh, Pearson, Ropar \& Hamilton, 2015; Nadig, Lee, Singh, Bosshart \& Ozonoff, 2010; Parish-Morris, Chevallier, Tonge, Letzen, Pandey \& Schultz, 2013). Research is also divided in regards to how social attention is distributed between different areas of interest (AOIs) of the social stimuli, with, for example, some studies showing increased attention to the mouth (Grossman, Steinhart, Mitchell, \& McIlvane, 2015; Jones et al., 2008; Klin et al., 2002) other showing no differences in attention to the mouth compared to TD controls (Birmingham et al., 2011; Rutheford et al., 2008; Tenenbaum et al., 2014 etc.) and yet others showing reduced attention to the mouth in ASD (Rice et al., 2012; Sterling et al., 2008). Similar discrepancies have been recorded in relation to attention to the eyes, the face as a whole, and the body (e.g. Hanley et al., 2013; Klin et al., 2002; Rice et al., 2012, etc.)

Attention to the non-social aspects of the world or to the task as a whole have also been found to differ, in some studies, between ASD and TD controls (Klin et al., 2002; Kuhn et al., 2010, Rice et al., 2012), while other studies report no differences (Norbury et al., 2009).

To better understand what the entire body of eye-tracking research is suggesting, I conducted a series of meta-analyses examining data from 73 papers that used eye-tracking methods to compare individuals with ASD and TD controls. The goal for this meta-analysis was to search for quantitative answers to the following questions:

1) Do individuals with ASD show overall diminished social attention?

2) What are the factors that affect how they distribute their attention between social and non-social stimuli?

3) Do individuals with ASD distribute their attention atypically between the different AOIs of social stimuli?

4) Do individuals with ASD engage more with the nonsocial stimuli than TD individuals?

5) Do individuals with ASD show have a generalized attention deficit, which results in atypical engagement with the task when being tested?

To answer these questions, I computed the overall effect size, the standardized difference in means between ASD and TD individuals, in total looking time for different AOIs: social elements of a scene, eyes, mouths, faces, and the bodies. I also computed the overall effect size for total looking time at the screen during eye-tracking tasks and for the total looking time at the non-social elements of the scene. Finally, I examined the impact of eight factors on the difference in overall social attention between these two groups: age, non-verbal IQ matching, verbal IQ matching, motion, social content, ecological validity, audio input and attention bids.

Results show that individuals with ASD spend less time attending to social stimuli than TD controls, with a mean effect size of 0.55. Also, social attention in ASD was most impacted when stimuli had a high social content (showed more than one person). Individuals with ASD also spent less time attending to eyes than TD controls (mean effect size of 0.35), less time attending to the mouth (mean effect size of 0.25 ), less time attending to the face (mean effect size of 0.40 ), but more time attending to the body (mean effect size of -0.48). Also, individuals with ASD spent less time attending to the screen when being tested (mean effect size of 0.53) and more time attending to the non-social elements of the stimuli (mean effect size of -0.34). These meta-analyses provide an opportunity to survey the eye-tracking research on social attention in ASD and to outline potential future research directions, for example research of social attention in the context of stimuli with high social content.

\title{
Severe Respiratory Dysrhythmia in Rett Syndrome Treated with Topiramate
}

\author{
Natalija Krajnc \\ University Children's Hospital Ljubljana, Slovenia
}

\begin{abstract}
Rett syndrome is a neurodevelopmental disorder that manifests itself early in childhood, progresses with the evolution of characteristic clinical signs and symptoms and is confirmed by mutation in the methyl-CpG-binding protein 2 gene. Seizures are present in a majority of Rett patients. Respiratory dysrhythmia in the awake state is present in two thirds of patients, leading in some cases to severe non-epileptic paroxysmal events. There are no optimal treatment recommendations thus far. We have documented with the clinical case studies the electro-clinical correlation of severe respiratory dysrhythmia mimicking seizures in two Rett patients and effective treatment with topiramate. Topiramate has many mechanisms of action and is widely used for its antiepileptic action in the treatment of epilepsy in children and adults. The effect of topiramate on specific carbonic anhydrase subtypes could be the mechanism of action that influenced the respiratory dysrhythmia in our Rett patients. Our
\end{abstract}


clinical observation suggests that topiramate is potentially helpful in the treatment of severe respiratory dyshythmia in Rett syndrome, but systematic research in a larger number of patients is required to evaluate its benefit.

\title{
Altered Cholinergic Activity and Increased Neural Inflammation in Neonatal Mice Injected with the Aluminum Adjuvant
}

\author{
Lucija Tomljenovic ${ }^{1^{*}}$, Dan $\mathrm{Li}^{1}$, Yongling $\mathrm{Li}^{1}$ and Christopher A. Shaw ${ }^{1-3}$ \\ ${ }^{1}$ Dept. of Ophthalmology and Visual Sciences, University of British Columbia, Vancouver, British Columbia, Canada \\ ${ }^{2}$ Program in Experimental Medicine, University of British Columbia, Vancouver, British Columbia, Canada \\ ${ }^{3}$ Program in Neuroscience, University of British Columbia, Vancouver, British Columbia, Canada
}

\begin{abstract}
Autism is a neurobehavioral disorder characterized by immune dysfunction manifested in early childhood, a window of early developmental vulnerability where the normal developmental trajectory is most susceptible to xenobiotic insults. The scientific consensus is that gene-environment interactions play a key role in autism. However, the molecular mechanisms about how genes interact with environmental factors remain obscure. A common xenobiotic with immuno-stimulating and neurotoxic properties to which infants worldwide are routinely exposed is the aluminum vaccine adjuvant. To investigate how aluminum interacts with genes involved in immune and nervous system function, we examined the expression of 17 genes which are implicated in both autism and innate immune response in brain samples of aluminum-injected mice. Some of the key players of innate immunity, such as CCL2, IFN $\gamma$ and $\mathrm{TNF} \alpha$, were significantly up-regulated, while the NF- $\kappa \mathrm{B}$ inhibitor NFKBIB, and acetylcholinesterase (ACHE), were down-regulated in the aluminum-injected male mice compared to control mice. It thus appears that aluminum triggered innate immune system activation and impairment of cholinergic activity in the male mice, observations which are consistent with those in autism. Female mice were less responsive to aluminum, as only expression levels of NFKBIB and TNF $\alpha$ were altered. Regional patterns of gene expression also exhibited sex differences: frontal cortex was the most affected area in males; cerebellum in females. Altogether, these results suggest that aluminum can impair brain function by penetrating the brain and interacting with neural and immune system mediators and that males are more susceptible to this type of aluminum toxicity.
\end{abstract}

\section{Roles of the Actin-Binding Protein Girdin in Neurodevelopment in Man and Mouse}

\author{
Masahide Takahashi", Atsushi Enomoto, Masato Asai and Naoya Asai \\ Dept. Pathology, Nagoya University Graduate School of Medicine, Japan
}

\begin{abstract}
We previously identified Girdin as a substrate for Akt kinase and an actin-binding protein. The protein consists of 1870 or 1871 amino acids that has an $\mathrm{N}$-terminal domain, a long coiled-coil domain and a C-terminal domain. The CT domain has the Akt phosphorylation site (ser-1416) and the actin-binding site. Our studies clearly showed that Girdin plays crucial roles in actin organization and cell migration in cultured cells and neuroblasts. Although Gidin $\mathrm{KO}$ mice are not embryonic lethal and are born at a Mendelian ration without any growth abnormalities, they died 3-4 weeks after birth and showed abnormal architectures in particular regions of the brain. The major phenotypes observed in Girdin $\mathrm{KO}$ mice were a defect in neuroblast migration born from neural stem cells in the subvetricular zone towards the olfactory bulb (OB), leading to OB hypoplasia, and mispositioning of immature neurons in the dentate gyrus (DG), resulting in the dispersion of the granular cell layer of the DG. The KO mice also had mesial-temporal lobe epilepsy, microcephaly and corpus callosum deficiency. Recently, a mutation in the Girdin protein was found in the British families with the PEHO (Progressive encephalopathy, with oedema, hypsarrythmia and optic atrophy) phenotype, indicating a crucial role of Girdin in human neurodevelopment. In addition, we generated Girdin knock-in mice (termed SA mice) in which serine 1416 (Akt phosphorylation site) was replaced with alanine. Although SA mice did not exhibit any anatomical or cytoarchitectural abnormalities in the brain, they showed significant impairment of long-term memory but not short-term memory. Our studies demonstrate that Girdin phosphorylation regulates the maintenance of LTP by linking the BDNF/TrkB signaling pathway with NMDA receptor.
\end{abstract}




\title{
Somatosensory Evoked Potentials in Children with Autism
}

\author{
Hanan Galal Azouz, Mona Khalil, Hayam Moustafa Abd EL Ghani and Hatim Mohamed Hamed \\ Alexandria University, Egypt
}

\begin{abstract}
Introduction: Autism is neurodevelopmental disorder which is characterized by widespread abnormalities of social interactions, communication, and severely restricted interests and highly repetitive behavior. Children with autism show sensory and perceptual abnormalities. They have either hyposensitivity or hypersensitivity to sensory, auditory, and visual stimuli.
\end{abstract}

Objectives: The aim of this work was to study somatosensory evoked potentials (SSEPs) changes among children with autism, and their relation to somatosensory manifestations and severity of autism.

Subjects: Thirty children with autism aged 2-12 years were included in the study, all of them fulfilling criteria of the Diagnostic and Statistical Manual of Mental Disorders (DSM-IV-TR).

Methods: All cases were subjected to thorough history taking including autistic symptoms and sensory abnormalities, comprehensive medical examination, psychiatric assessment according to DSM-IV-TR criteria for diagnosing autism, assessment of severity of autism using Childhood Autism Rating Scale (CARS) and measurement of somatosensory evoked potentials elicited by median nerve stimulation at wrist.

Results: The majority of the cases were males (86.7\%), according to CARS $53.3 \%$ were classified as mild to moderate autism, while $46.7 \%$ were severe. Sensory abnormalities were present in $56.7 \%$ of cases. Somatosensory abnormalities were present in $36.76 \%$ of the cases. There was statistical significant relationship between sensory symptoms with SSEP abnormalities. $(\mathrm{P}=0.040)$. The presence of abnormal SSEPs was not statistically associated with higher score in CARS.

Conclusions: Children with autism have an abnormal SSEP changes and were significantly related to the presence of sensory abnormalities, indicating central cortical dysfunction of somatosensory area. On the other hand, these abnormal SSEP changes were not related to the severity of autism.

\section{Sex Differences in Rodent Models of Neonatal Hypoxia Ischemia}

\author{
Jaylyn Waddell ${ }^{*}$ and Margaret M. McCarthy ${ }^{2}$ \\ ${ }^{1}$ Department of Pediatrics, University of Maryland, Baltimore, School of Medicine, Baltimore, MD, USA \\ ${ }^{2}$ Department of Pharmacology, University of Maryland, Baltimore, School of Medicine, Baltimore, MD, USA
}

\begin{abstract}
Hypoxia ischemia (HI) of the brain in infants remains a leading cause of mortality and lifelong disability. Males exhibit more severe impairments than females in both human and animal models. In a series of histological and behavioral assays, we have captured this sex difference using a modification of the Rice-Vannucci model of unilateral carotid artery ligation followed by exposure to low oxygen conditions at postnatal days 7 and 10. Male rats subjected to HI are more generally impaired in simple reflex and memory tests than females. Converging evidence from our research confirms that while HI-elicited damage in the female brain appears to be restricted to the hemisphere ipsilateral to the carotid artery ligation, the male brain is more generally affected. Volume loss is detected bilaterally in males compared to same-sex sham operated controls. Males and females do not differ in the volume of the lesion ipsilateral to the ligation. Further, we find that putative repair mechanisms, such as neurogenesis favor the female brain, and do so bilaterally following injury. Our data emphasize the importance of potential sex differences in diaschisis following perinatal brain injury. Quantification of lesion volume, or use of the contralateral hemisphere as a comparative control might neglect important global changes in brain that contribute to the long observed vulnerability of males to $\mathrm{HI}$ relative to females.
\end{abstract}




\title{
A Machine Learning Paradigm for Ultrasonic Echolucent Plaque Characterization for Carotid Near and Far Walls - Stroke Risk Stratification
}

\author{
Tadashi Araki ${ }^{1}$, Pankaj K. Jain ${ }^{2}$, Harman S. Suri ${ }^{3}$, Narendra D. Londhe ${ }^{2}$, Nobutaka Ikeda ${ }^{4}$, Ayman El-Baz ${ }^{5}$, Luca Saba ${ }^{6}$, \\ Andrew Nicolaides ${ }^{7,8}$, Shoaib Shafique9, John R. Laird ${ }^{10}$, Ajay Gupta ${ }^{11}$ and Jasjit S. Suri ${ }^{11-13^{*}}$ \\ ${ }^{1}$ Division of Cardiovascular Medicine, Toho University Ohashi Medical Center, Tokyo, Japan \\ ${ }^{2}$ Department of Electrical Engineering, NIT Raipur, Chhattisgarh, India \\ ${ }^{3}$ Monitoring and Diagnostic Division, AtheroPoint ${ }^{T M}$, Roseville, CA, USA \\ ${ }^{4}$ Cardiovascular Medicine, National Center for Global Health and Medicine, Tokyo, Japan \\ ${ }^{5}$ Department of Bioengineering, University of Louisville, KY, USA \\ ${ }^{6}$ Department of Radiology, University of Cagliari, Italy \\ ${ }^{7}$ Vascular Screening and Diagnostic Centre, London, England \\ ${ }^{8}$ Vascular Diagnostic Centre, University of Cyprus, Nicosia, Cyprus \\ ${ }^{9}$ CorVasc Vascular Laboratory, 8433 Harcourt Rd \#100, Indianapolis, IN, USA \\ ${ }^{10}$ UC Davis Vascular Centre, University of California, Davis, CA, USA \\ ${ }^{11}$ Brain and Mind Research Institute and Department of Radiology, Weill Cornell Medical College, NY, USA \\ ${ }^{12}$ Point-of-Care Devices, Global Biomedical Technologies, Inc., Roseville, CA, USA \\ ${ }^{13}$ Department of Electrical Engineering, University of Idaho (Aff.), ID, USA
}

\begin{abstract}
Atherosclerotic disease is a multifocal disease and not restricted to one of the walls of the carotid artery. Grayscale morphology of the ultrasound carotid wall has recently been shown to have a promise in stroke risk stratification for high risk versus low risk plaque or symptomatic versus asymptomatic plaques. Previous studies have shown characterization for only the far wall ultrasound of the carotid artery. This study presents a new approach for stroke risk assessment by integrating assessment of both the near and far walls of the carotid artery using grayscale morphology of the plaque. Further, this paper presents a scientific validation system for stroke risk assessment. The system first segments the near and far walls of the carotid artery in B-mode US scans. The feature extraction system consisted of sixteen grayscale texture features which are then fed into the machine learning system. The training system utilizes the carotid stenosis criteria as ground truth labels for the stratification of stroke risk. The cross-validation procedure is adapted in order to obtain the machine learning testing stratification accuracy using three sets of partition protocols: $(K=5, K=10$, and Jack Knife). The mean stratification accuracy for the automated system corresponding to all three protocols in the far and near walls is $95.08 \%$ and $93.47 \%$, respectively. The corresponding accuracies for the benchmarking manual system are $94.06 \%$ and $92.02 \%$, respectively. The precision of merit of the automated system when compared against manual risk assessment system are $98.05 \%$ and $97.53 \%$ for the far and near walls, respectively. The ROC of the risk assessment system for the far and near walls is close to 1.0, demonstrating high accuracy. The pre-commercial version shows promising results.
\end{abstract}

\section{Disruption of Superoxide-Stimulated Glutamate Release in Ischemic Penumbra as a New Viable Strategy for Neuroprotection in Stroke}

\author{
Alexander A. Mongin", Preeti Dohare, Aarshi Vipani, Nicole H. Bowens, María C. Hyzinski-García, Paul J. Feustel, David \\ Jourd'heuil and Richard W. Keller Jr.
}

Albany Medical College, Albany, NY, USA

\begin{abstract}
Antioxidant agents potently protect against ischemic brain damage in animal models, but have shown little to no clinical benefits in humans. The reasons for failure of the antioxidant strategies in human stroke are not clear. Recently we found that, unlike other antioxidants, the superoxide dismutase mimetic tempol strongly reduces intra ischemic release of the excitotoxic neurotransmitter glutamate in a rodent model of stroke (P. Dohare et al. Free Radical Biol. Med. 77: 168, 2014). Effects of tempol and other antioxidants on pathological glutamate release were quantified in the ischemic penumbra before, during and after 2-hr occlusion of the middle cerebral artery, using a microdialysis approach. Reductions in glutamate release correlated with decreased brain infarction volumes and improvements on several behavioral outcomes, suggesting new glutamatedependent mechanism of neuroprotection. In contrast to tempol, other "typical" antioxidants, such as edaravone (Radicut $\left.{ }^{\circledast}\right)$, were much less effective. We further explored molecular nature of the redox-sensitive glutamate release in the penumbra utilizing the combination of micro dialysis and a laser Doppler velocimetry. Pharmacological analysis identified two new glutamate
\end{abstract}


permeability pathways: volume-regulated anion channels (VRAC), which are permeable to glutamate, and the $\mathrm{Ca}^{2+}$-dependent increases in membrane glutamate leak. VRAC is thought to mediate the onset of glutamate release in penumbral tissue and drive propagation of stroke injury. Altogether, our work sheds light on novel mechanisms of oxidative damage in stroke, and suggests that one class of the antioxidants - SOD mimetics - may have unique and potent neuroprotective properties due to their effects on glutamate-driven brain damage in the ischemic penumbra.

\title{
Reversing Abnormal Hypertonia in Post-stroke Patients using the Neuro-IFRAH ${ }^{\circledR} /$ Al-Oboudi Approach
}

\author{
Maria Amelia Chang \\ Neuro-IFRAH Organization, San Diego, CA, USA
}

\begin{abstract}
It is known that post-stroke spasticity or hypertonia can be managed by conservative measures. To be clinically relevant, the Neuro-IFRAH/ Al-Oboudi approach use the term, abnormal muscle activity that can be changed by intervention instead of poststroke spasticity. The following case studies show the reversal of abnormal muscle activity in individuals affected by stroke after treatment from occupational therapist, Waleed Al-Oboudi originator of the Neuro-IFRAH Approach. This study emphasizes clinical expertise in applying specific rehabilitative interventions necessary for the acquisition of close to normal movement. The 2 case studies demonstrate the potential for recovery extends to individuals with chronic post-stroke spasticity or hypertonia. Both patients were treated in a clinic by the author of the Neuro-IFRAH Approach, Al-Oboudi, an occupational therapist and international educator in rehabilitation of individuals affected by stroke or brain injury. These studies emphasize that clinicians must be able to design interventions and show results beyond the impairment level; specific interventions that progressively and continually support the regained motor recovery for the patient to be able to participate in functional activities and their life roles. Thus, the importance of clinical expertise in applying effective interventions and understanding the process of rehabilitation is key to activity dependent neuroplasticity. Neuroscientists, academic researchers and clinicians in the field of post-stroke rehabilitation need to consider the following: 1. Poorly applied therapeutic interventions and erroneous delivery of rehabilitative add to the complex pathophysiology and clinical features of spasticity in post-stroke patients; 2 . Non-skilled clinicians will not be able to reverse abnormal hypertonia.
\end{abstract}

\section{The Novel Optokinetic Chart Stimulation as a Stroke Recovery Intervention}

\author{
Benjamin Chitambira \\ East Kent Hospitals University NHS Foundation Trust, United Kingdom
}

\begin{abstract}
Background and Purpose: Optokinetic chart stimulation (OKCS) is evolving as a stroke and neuro-recovery rehabilitation intervention for completely paralysed patients who pose a huge cost to the health and social care economy. The aim is to show results for patients who benefit from OKCS.

Methodology: The optokinetic chart is made of laminated A4 paper and consists of repeated colours of the rainbow. From 20 centimeters in front of a patient's face, the chart is moved from side to side at one cycle per second for 3 minutes. The chart is then moved up and down for 3 minutes and then forwards and backwards for 3 minutes.

Results: With the OKCS based OKCSIB protocol, recovery from complete paralysis occurs provided the acute strokes are parietal, basal ganglia, fronto-parietal, parieto-occipital or brain stem. A retrospective case controlled series showed that upper limbs recovered better with the OKCSIB protocol. A prospective randomised case controlled study saw the completely hemiplegic experimental patient fully recover voluntary movements whilst the control who received usual care did not recover at all but ended up bed bound and spastic. Two bed bound chronic strokes with hemiplegia recovered lower limb movements and assisted mobility after OKCS.
\end{abstract}

Conclusion: OKCS shows preliminary efficacy in completely paralysed acute strokes and chronic strokes. Randomised controlled trials are needed to provide evidence for its wider uptake in clinical practice. 


\title{
Twenty-yearTrends in Mortality Rates from Stroke in Klaipeda
}

\author{
Henrikas Kazlauskas $^{{ }^{*}}$, Nijole Raskauskiene ${ }^{2}$, Rima Radziuviene ${ }^{2}$ and Vinsas Janusonis ${ }^{1}$ \\ ${ }^{1}$ Klaipeda University Hospital, Lithuania \\ ${ }^{2}$ Behavioral Medicine Institute Lithuanian University of Health Sciences, Lithuania
}

\begin{abstract}
Methods: Data on permanent residents of Klaipeda (Lithuania) aged 35-79 years who died from stroke in the period of 1994 to 2013 were gathered. Directly age standardized (European population) stroke mortality rates were calculated and analyzed using joinpoint regression separately for specific age groups (35-64, 65-79, 35-79) and by gender. Annual percentage change (APC) and 95\% CIs were presented.

Results: Age-adjusted (European standard population) stroke mortality rates declined by 35\% in men and 63\% in women in Klaipeda 1994-2013. Stroke mortality peaked in the years 1994-1997, and began to decrease after that time by $-9.9 \%(95 \%$ CI: -48.7 to -0.2 ) yearly until 2001 and leveled off between 2001 and 2013. Among 35-64 years old men, the mortality rate decreased by $12.8 \%$ (-21.5 to -3.3) yearly from 1994 to 2001 and turned positive by $6.3 \%$ (0.8 to 12.1) between 2000 and 2013 . Among 35-64 years old women, mortality decreased significantly by $15.5 \%$ (-28.1 to -3.3 ) from 1994 to 2000 , and there was evidence of recent plateauing of trends between 2000 and 2013. A steady decline in mortality continued among both sexes aged 65-79 years old: mortality significantly decreased from 1994 onwards by $-5.5 \%(-7.9$ to -3.0$)$ yearly in women and by $-3.3 \%$ $(-5.6$ to -0.9$)$ in men.
\end{abstract}

Conclusions: There is evidence that stroke mortality rates among 35-64 years old men and women have been more likely to plateau in the first decade in new millennium. Stroke mortality rates declined incessantly between 1994 and 2013 among both sexes aged 65-79 years.

\section{Ischemic Stroke at Jordan University Hospital: A One-year Hospital-based Study of Subtypes and Risk Factors}

\author{
Bahou $Y^{*}$, Ajour $M$ and Jaber $M$ \\ Jordan University Hospital, Amman, Jordan
}

\begin{abstract}
Objective: To study the ischemic stroke subtypes and risk factors in 100 patients observed at Jordan University Hospital (JUH) over a one-year-period, and to compare the results with another 100 age-and -sex matched controls as well as with studies from other Arab countries.

Methods: One hundred patients with first-ever ischemic stroke admitted to JUH over a one-year period (between January 2012 and January 2013) were studied.

Results: There were 62 males and 38 females $(\mathrm{M} / \mathrm{F}$ ratio $=1.6)$, with a mean age of 66 years (range 22-90 years), the majority (80/100) between the age 51-80 years. The most common stroke subtype was lacunar infarcts (36 patients). Fourtytwo out of 51 patients had intracranial atherosclerosis. The most common risk factor was hypertension (85\%) followed by hyperlipidemia (71\%) and diabetes mellitus (65\%).

Conclusion: In accordance with other Arab studies and controls, hypertension was the predominant risk factor but lacunar infarcts were more common than in most reports from other Arab countries. This shows the importance of appropriate management of hypertension to reduce the incidence of stroke in Jordan.
\end{abstract}




\title{
Multiple Sclerosis and Suppressor of Suppressor of Cytokine Signaling (SOCS) Proteins
}

\author{
Virginia Sedeño Monge ${ }^{1 *}$, Raul Arcega Revilla ${ }^{2}$, Emanuel Rojas Morales ${ }^{3}$, Gerardo Santos López ${ }^{4}$ and Julio Reyes Leyva ${ }^{4}$ \\ ${ }^{1}$ Departamento de Ciencias de la Salud, Universidad Popular Autónoma del Estado de Puebla, México \\ ${ }^{2}$ Servicio de Neurología, Instituto Mexicano del Seguro Social, Mexico \\ ${ }^{3}$ Maestria en Biotecnología, Universidad Popular Autónoma del Estado de Puebla, Puebla, Mexico \\ ${ }^{4}$ Laboratorio de Biología Molecular y Virología, Centro de Investigación Biomédica de Oriente, Instituto Mexicano del Seguro Social \\ Puebla, México
}

\begin{abstract}
Multiple Sclerosis (ME) is a demyelinating, chronic and autoimmune disease of the Central Nervous System. Different treatments have been used; between them IFN-b, glatiramer acetate (AG), and natalizumab, however the disease progresses; and only $30 \%$ of patients are considered as responders to some treatments (IFN-b and GA). The efficiency and adverse effects are specific of each therapy, the way that these modulate the immune response is important to elucidate the consequent improvement in the patients. Some cytokines like IFN-g have been identified in the pathogenesis of ME. Some members of family of suppressors of cytokine signaling (SOCS) proteins regulate and are activated by proinflammatory cytokines implicated in ME; SOCS1 and SOCS3 (both proteins regulators of JAk-STAT pathway, initiated by cytokine as IFN-g) appear be implicated in the pathophysiology of ME. In a previous study we found an increased transcription of SOCS3 and a decreased transcription in SOCS1 in patients with ME in comparison with healthy subjects. Other members of SOCS family are implicated in autoimmune disease, SOCS7 inhibits IL-23 and IL-6, both proinflammatories cytokines, in other hand SOCS5 has been studied in autoimmune disease. Currently we are studying transcription levels of SOCS5 and SOCS7 in patients with ME, and we are finding that transcription level of SOCS5 and SOCS7 are increased in patients with ME in comparison with healthy individuals. The study of SOCS family is important to elucidate the immunological basis of ME pathogenesis.
\end{abstract}

\section{Citicoline: Myelination-improving Food Supplement}

\section{Paweł Grieb}

Department of Experimental Pharmacology, Mossakowski Medical Research Centre, Polish Academy of Sciences, Warsaw, Poland

\begin{abstract}
Although food supplements by definition are to be added to the diet to benefit health thanks to their nutritional or physiologic effects, few if any are considered really useful. For several reasons citicoline (cytidine-5'-diphosphocholine) may be unique in this respect. Being a natural compound ubiquitously present in all cells where it acts as an intermediate in phospholipid synthesis, for many years citicoline was an injectable nootropic and neuroprotective prescription drug. Later it has been shown to reinforce brain phospholipids as well when given orally. Although it has gained considerable popularity in some countries, two recent large phase 3 studies failed to prove its efficacy in acute stroke and traumatic brain injury. In the meantime, taking into consideration that it is apparently devoid of any toxic effects, citicoline has been reclassified and became a food supplement and a dietetic choline source both in USA and the European Community. Numerous studies have indicated that citicoline is able to reinforce some cns neurotransmitter systems (eg. dopamine), enhance cellular membrane repair, attenuate glutamate excitotoxicity, retard senescence of brain mitochondria, mimic some neurotrophic factors, etc. One particularly interesting effect of citicoline may be its ability to counteract demyelination and reinforce remyelination, which has been shown with preclinical models of demyelination and EAE. These effects may have relevance to multiple sclerosis, and they also may provide explanation to memory enhancement by citicoline reported both in animals and humans.
\end{abstract}

\section{Neurocysticercosis: An Emerging Public Health Problem}

\author{
Kashi Nath Prasad \\ Sanjay Gandhi Postgraduate Institute of Medical Sciences, Lucknow, India
}

\section{Abstract}

Neurocysticercosis (NCC) is identified as the most common cause of acquired active epilepsy (AE) in the developing countries. 
$\mathrm{NCC}$ is an emerging problem worldwide because of increased human travel and migration of Taenia solium carriers/ infected subjects. We investigated NCC burden in pig farming community of North India. Since many NCC infected individuals remain asymptomatic, we further investigated host genes polymorphisms and their relation with NCC related seizure disorders. Total 294 families with 1640 subjects from 30 villages were surveyed for AE. All individuals with AE and 595 asymptomatic subjects underwent magnetic resonance imaging of the brain. TLR4, MMP9, ICAM1 and GST genes polymorphisms and their expressions were studied to define their role in symptomatic disease. Slaughtered pigs were screened for cysticercosis. Total 95 (5.8\%) subjects with AE were identified; 48.3\% of them had NCC. Epilepsy in the family and no separate place for pig were identified as risks for NCC clustering. Treatment gap for AE in the community was above 90\%. Ninety (15\%) asymptomatic individuals also had NCC. When compared between symptomatic and asymptomatic NCC subjects, polymorphic TLR4, MMP9, ICAM1 and GST genotypes with enhanced expressions were identified as risks for development of seizure disorders. Thirteen (26\%) of 50 pigs slaughtered had cysticercosis. The results showed high NCC burden in pig farming community and NCC as major cause of AE. Individuals with polymorphic TLR4, MMP9, ICAM1 and GST genotypes were susceptible for symptomatic disease. High swine cysticercosis prevalence suggests the transmission dynamic between human and swine in the community.

\title{
Smoke and Mirrors: Limited Value of Relative Risk Reductions for Assessing the Benefits of Disease-modifying Therapies for Multiple Sclerosis
}

\author{
Magd Zakaria \\ Faculty of Medicine, Ain Shams University, Cairo, Egypt
}

\begin{abstract}
The relative risk reduction (RRR) is the main statistical parameter used to express the different primary outcomes of the clinical drug trials. Physicians often assume that a drug with a higher RRR demonstrated in one trial is more effective than a drug with a lower RRR demonstrated in another trial, and may pass this idea on to younger physicians and to the patients. The use-of the RRR as a measure of drug efficacy can be misleading as it depends on the nature of the population studied. The value of the RRR depends on the placebo event rate: A low RRR can be clinically meaningful if the event rate in the placebo group is high, while a high RRR can be clinically less meaningful if the event rate in the placebo group is low. Direct head to head comparison trials are the only way to assess the relative efficacy of the different drugs. The aim of this presentation is to correct this misconception.
\end{abstract}

\section{Kennedy Disease and Sex Hormones}

\author{
Jin Jun Luo ${ }^{1,2^{*}}$ and Nae J. Dun ${ }^{2}$ \\ ${ }^{1}$ Departments of Neurology, Lewis Katz School of Medicine at Temple University, Philadelphia, PA, USA \\ ${ }^{2}$ Departments of Pharmacology, Lewis Katz School of Medicine at Temple University, Philadelphia, PA, USA
}

\begin{abstract}
Kennedy disease (KD), or spinobulbar muscular atrophy, is an X-linked neuromuscular disorder caused by a mutation in the androgen receptor gene, resulting in an expansion of CAG trinucleotide repeats. Clinically, it is characterized by muscle weakness, atrophy, and fasciculation. Facial, particularly peri-oral, fasciculation is an early characteristic feature. Dysphonia and dysphagia may also occur. Tendon reflexes are usually decreased. Sensation may be decreased. Gynecomastia, testicular atrophy, and reduced fertility are caused by androgen receptor dysfunction. Electrodiagnostic study typically shows sensory axonal neuropathy and chronic neurogenic changes. A mutation in the androgen receptor gene appears to be unique to KD. Diagnosis is confirmed by polymerase chain reaction showing abnormal expansion of CAG repeats. Recently much effort has been made in clinical and basic research relative to the pathogenesis of sex hormones in the development of Kennedy disease and possibly therapeutic strategies. We present our review on the update views in this regard.
\end{abstract}

\section{Reduced Interleukin 7 Receptor Alpha Gene Expression in Multiple Sclerosis Patients- A Study in Egypt}

\section{Shahira Elshafie*, Howaida Abdel Rasool, Hanan Helmy and SherifYounan}

Fayoum University, Egypt 


\begin{abstract}
Multiple sclerosis has a clinically significant heritable component. The interleukin 7 receptor alpha (IL-7RA) has been recognized as a susceptibility gene for multiple sclerosis (MS). It is known that demographic, environmental factors, as well as population genetic background have a substantial role in multiple sclerosis development. The aim of the present study was to assess the relevance of IL-7RA messenger RNA (mRNA) gene expression level in peripheral blood mononuclear cells (PBMC) on MS phenotype (including clinical and magnetic resonance imaging (MRI) parameters). A total of 31 unrelated Egyptian patients with MS compared to 14 unrelated matched healthy controls were included in the study. IL-7RA VEGF-A gene expression and glyceraldehyde-3-phosphate dehydrogenase (GAPDH) as a housekeeping gene were measured by real-time polymerase chain reaction (RT-PCR) using SYBR Green technique. IL-7RA mRNA gene expression level was significantly lower in the MS group compared to the control group ( $\mathrm{p}<0.001)$. Reduced mRNA IL-7RA expression was observed both in patients with MS compared to the control group and in stratified analysis of MS subtypes: primary progressive MS (PPMS), relapsing progressive MS (RPMS), and secondary progressive MS (SPMS). IL-7RA mRNA gene expression level was not significantly different in MS patients in relapse compared to those in remission $(p>0.05)$. There was no statistically significant difference in IL-7RA mRNA gene expression level among MS patients with MRI $\geq 9$ brain lesions compared to MS subjects with MRI < 9 brain lesions ( $p>0.05$ ). IL-7RA mRNA gene expression level cannot be used as a stratifying tool in determining disease course. There was no correlation between IL-7RA mRNA gene expression levels and neither age, age of onset, duration of disease, multiple sclerosis progression index, nor expanded disability status scale. These results confirm the involvement of mRNA gene expression of IL-7RA in MS pathogenesis and suggest that IL-7RA variation may primarily affect chronic disease course.
\end{abstract}

\title{
The Roles of NeurotransmitterFunction and Gut Health in BipolarDisorder:Functional Clinical Approaches for Modulation and Treatment
}

\author{
Cicely R. Everson ${ }^{1-3}$ \\ ${ }^{1}$ Tao Holistics, USA \\ ${ }^{2}$ American Academy of Anti-Aging Medicine, FL, USA \\ ${ }^{3}$ Functional Medicine University, SC, USA
}

\begin{abstract}
Bipolar Disorder (BP) is one of the most common neurological disorders present today. This progressive condition is characterized pathologically by cycles of depression, contrasted with emotional highs, or manic states. The understanding of the neurochemical mechanisms underlying this pathology has been recognized, yet rarely correlated with the mitigation of BP symptomology. Here, we review the underlying neurochemical mechanisms of Bipolar Disorder and consider what might be clinically effective in eliminating the symptomology indicative of both depressive and manic states of this condition. Data from patient samples were used to display a unique clinical approach into the modulation of these symptoms. Based on current data and clinical findings, I explain how nutritional deficiencies, poor gut function and hormonal imbalance cumulatively contribute to the neuropathogenesis of Bipolar Disorder. I also provide an effective, therapeutic protocol for treatment.
\end{abstract}

\section{Drugging the Undruggable: Insights from Huntington's Disease}

\author{
Boxun Lu \\ School of Life Sciences, Fudan University, Shanghai, China
}

\begin{abstract}
Most neurodegenerative disorders are associated with accumulation of disease-relevant proteins. Among them, Huntington Disease (HD) is an appealing model because of its monogenetic nature. HD is caused by the mutation of the Huntingtin (HTT) gene, which encodes the mutant HTT protein (mHTT) with an expanded polyglutamine tract. mHTT cytotoxicity is the major cause of the disease, and thus lowering mHTT level is a promising treatment strategy. Here I will discuss several studies from my lab pursuing this strategy using genetic screening and validation approaches, as well as several validated targets have validated in vitro and in vivo, serving as entry points for HD drug discovery.
\end{abstract}




\title{
Seizure Reporting Technologies for Epilepsy Treatment: A Review of Clinical Information Needs and Supporting Technologies
}

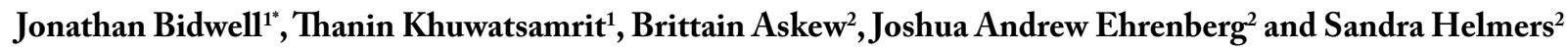 \\ ${ }^{1}$ School of Interactive Computing, Georgia Institute of Technology, Atlanta, GA, USA \\ ${ }^{2}$ School of Medicine, Emory University, Atlanta, GA, USA
}

\begin{abstract}
Mobile and wearable technologies are becoming increasingly capable of seizure detection and reporting. This review surveys current seizure detection and classification technologies as they relate to aiding clinical decision-making during epilepsy treatment. Interviews and data collected from neurologists and a literature review highlighted a strong need for better distinguishing between patients exhibiting generalized and partial seizure types as well as achieving more accurate seizure counts. This information is critical for enabling neurologists to select the correct class of antiepileptic drugs (AED) for their patients and evaluating AED efficiency during long-term treatment. In our questionnaire, $100 \%$ of neurologists reported they would like to have video from patients prior to selecting an AED during an initial consultation. Presently, only $30 \%$ have access to video. In our technology review we identified that only a subset of available technologies surpassed patient self-reporting performance due to high false positive rates. Inertial seizure detection devices coupled with video capture for recording seizures at night could stand to address collecting seizure counts that are more accurate than current patient self-reporting during day and night time use.
\end{abstract}

\section{The Potential Promise of Immunotherapy in CNS Cancers}

\author{
Deepa Subramaniam \\ Georgetown University, Washington, DC, USA
}

\begin{abstract}
Notions of the central nervous system as an immune-privileged site have long been held since early 20th century. More recent evidence suggests that this may not be true and that there is an active immune surveillance system with glial cells themselves functioning as antigen-presenting cells, and lymphatics draining via the cribriform sinus into cervical lymph nodes, thus allowing immune cell transit despite the tightly guarded blood-brain barrier. However, brain tumors especially glioblastoma, may continue to be immune suppressive via expression of indoleamine-2,3-dioxygenase (IDO) and transforming growth factor- $\beta$ (TGF- $\beta$ ) which helps to recruit immunosuppressive regulatory T cells, Phosphatase and Tensin Homolog (PTEN) loss which can up regulate programmed death ligand-1 (PDL-1) expression and down regulation of major histocompatibility complex (MHC) and B7 family of proteins.

A number of immunotherapeutic approaches have been explored in neuro-oncology. Initial efforts attempted to deliver toxins as diphtheria or pseudomonas toxins to the tumor tagged with either radionucleotides (I131) or interleukins. Other efforts have used vaccines, either peptide based vaccines or dendritic cell vaccines created by exposing freshly harvested tumor antigens to peripheral blood monocytes. Oncolytic viral therapy using herpes simplex virus, adenovirus or polio virus to cause direct tumor cell lysis and immune recruitment for ongoing anti-tumor activity are being explored actively. Most recently, the success of immune checkpoint blockade in solid tumors has allowed its exploration in high grade gliomas, with antiprogrammed death 1 (anti-PD1) and anti-cytotoxic T-lymphocyte antigen 4 (anti-CTLA4) antibodies currently in clinical trials for glioblastoma and brain metastases especially in melanoma.
\end{abstract}

\section{Glowing Gliomas - New Tricks with Fluorescence Guided Resection of CNS Tumors}

\section{Brent T. Harris}

Departments of Pathology and Neurology, Georgetown University School of Medicine, Washington, DC, USA

\begin{abstract}
Neurosurgeons are faced with the daunting challenge of resecting CNS neoplasms to improve patient symptoms and survival and to obtain tissue for diagnosis to guide further treatment. At the same time, they are trying to minimize resection of normal CNS tissues that could leave the patient with neurologic deficits. Modern imaging and navigation systems allow the surgeon
\end{abstract}


to plan and carry out in 3 dimensions optimal resections. However, these are still lacking given the limitations of pre-operative imaging and tissue shifting during surgery. Fluorescence guided resection techniques enable direct visualization of pathological tissues during surgery allowing the surgeon to make critical decisions about what to resect as the operation proceeds. Here we discuss data from our ongoing clinical trial to investigate the utility of pre-surgical administration of 5-aminolevulinic acid and visualization and quantification of protoporphyrin 9 in a variety of CNS neoplasms.

\title{
Migraine Mythology 101 - The Facts are Far More Exciting Than the Fiction
}

\section{P. Christopher H. Gottschalk}

Yale University School of Medicine, CT, USA

\begin{abstract}
There has been an explosion of knowledge in the field of Headache Medicine in the first part of the 21st century. We now have detailed physiological models of primary headaches (migraine, cluster, thunderclap) that inform diagnosis and treatment in exciting new ways. However, the bulk of new ideas regarding recognition and management of these disorders remains sadly obscure to most physicians, neurologists included. A major obstacle to the dissemination of these concepts is the persistence of old, unsubstantiated and more often disproven, ingrained ideas-i.e., mythology. The notion that migraine is a "vascular" headache or that "tension" and "stress" are important causes of head pain must be discarded in order to recognize and treat headache disorders effectively and appropriately; even the concept of "triggers" that need to be managed is refuted by the emerging evidence of the nature and duration of the premonitory phase of migraine, or prodrome. This presentation aims to identify and dissect those persisting myths that interfere with proper assessment and treatment of the most prevalent and disabling disorders in the general population.
\end{abstract}

\section{Headache: It's All in your Head...or is it?}

\author{
Paul G. Mathew ${ }^{1-4}$ \\ ${ }^{1}$ Brigham E Women's Hospital, Department of Neurology, John R. Graham Headache Center, Boston, MA, USA \\ ${ }^{2}$ Harvard Vanguard Medical Associates/Atrius Health, Department of Neurology, Braintree, MA, USA \\ ${ }^{3}$ Cambridge Health Alliance, Division of Neurology, Cambridge, MA, USA \\ ${ }^{4}$ Harvard Medical School, Boston, MA, USA
}

\begin{abstract}
Headache is one of the most common complaints among patients presenting for an outpatient neurology consultation. The evaluation and diagnosis of headache disorders according to the International Headache Society's International Classification of Headache Disorders, 3-Beta Edition will be discussed. Pharmacological and non-pharmacological interventions will be reviewed including lifestyle modifications with an emphasis on trigger avoidance. Injectable therapies and surgical interventions will also be discussed. The primary focus will be on migraine, but secondary headaches and other primary headache disorders will be briefly highlighted including trigeminal autonomic cephalalgias, tension-type headache, and cranial neuralgias.
\end{abstract}

\section{Pain Localization in Migraine without Aura and Cognitive Behavioral Correlates: The Neurophysiological and Anatomo-functional Perspective}

\author{
Marzia Buonfiglio*, Giuliano Avanzini and Francesco Di Sabato \\ Policlinico Umberto I, Sapienza University of Rome, Italy
}

\begin{abstract}
As of today, migraine etiology is still not completely understood, likewise the causal factor of pain localization has not yet been clarified. Recently we demonstrated, for the first time, a strong correlation between analytic cognitive style and both migraine and lack of habituation of evoked visual potentials. We aimed at investigating whether visual/auditory perceptual dimension of cognitive style may account for left $\backslash$ right localization of pain in migraine without aura (MWOA). We enrolled 150 MWOA patients at our headache center, and a control group of healthy volunteers. Using a scoring system, we categorized patients as having right or left unilateral pain localization. Utilizing cognitive behavioral test, we investigated analytic/global and
\end{abstract}


visual/auditory cognitive style of patients and healthy subjects. We found significant relationship between visual analytic style and left side unilateral MWOA patients, and significantly higher auditory analytic scores in unilateral right side migraineurs (ANOVA TEST, $\mathrm{p}<0.005$ ). We discuss our results in the light of our recent neurophysiological evidence obtained by evoked potentials techniques and from an anatomo-functional perspective suggesting a key role of cognitive behavior in migraine that may account also for pain localization. A potential clinical significance can be underlined because visual/auditory analytic information processing style can be modified by appropriate cognitive behavioral training indicating new potential therapeutic strategies for migraine.

\title{
The Psychiatric Comorbidities of Migraine
}

\author{
Mark W. Green
}

Icabn School of Medicine at Mt Sinai, New York, NY, USA

\begin{abstract}
Migraine is the most common variety of headache that prompts a visit to a physician for headaches. Migraine has many psychiatric comorbidities which can influence the headache and can confound the treatment of both migraine and the comorbidity. The talk explains the biological basis of migraine and how the psychiatric comorbidities are related and how treatment can be altered to deal with both disorders.
\end{abstract}

\section{The Clinical Significance of Voice and Swallowing Pathology in Parkinson's Diseases}

\author{
Zoltan Mari \\ Johns Hopkins University, School of Medicine, Baltimore, MD, USA
}

\begin{abstract}
Parkinson disease (PD) has long been simplified in "motor symptom centered" and "dopamine centered" approaches, which determined the prevailing ways how we viewed this prevalent and highly disabling neurodegenerative disease. More recently, however, we had to confront how insufficient such deductive vision was and the complexity of PD is now being increasingly recognized. PD patients of course do have dopamine deficit and many motor symptoms, but these hardly tell the whole story or even define the disease satisfactorily. In fact, non-motor and non-dopaminergic symptoms are often among the most disabling for patients. Speech impairment remains one of the most often reported such disability in PD. It is complex, the leading contributor to speech disability could be (1) cognitive impairment/language deficit; (2) impaired articulation (dysarthria); (3) low volume (hypophonia); (4) abnormally rapid speech or cluttering (tachyphemia); or (5) some combination and/or unknown abnormality. As common and as disabling speech impairment in PD may be, it is one of the most often overlooked or not adequately evaluated and treated source of disability. Unfortunately, some traditional treatment modalities (certain medications, DBS) are not only unhelpful, they could in fact exacerbate speech problems. Fortunately, several therapy approaches have been shown to be beneficial and should always be recommended, together with a detailed evaluation in the hands of an experienced speech professional. Dysphagia is often an even more dreaded than speech impairment, causing major concern for patient safety due to the high morbidity and even mortality risk of aspiration pneumonia. This is particularly clinically significant in advanced disease, but often gets overlooked and insufficiently addressed. In this overview, we discuss the importance of speech and swallowing impairments in $\mathrm{PD}$, along with a practical algorithm to optimize evaluation and treatment.
\end{abstract}

\section{Hypophonia in Parkinson's Disease: Medical and Surgical Considerations}

\author{
Lee M. Akst
}

Johns Hopkins Medical Institutes, Department of Otolaryngology-Head and Neck Surgery, Baltimore, MD, USA

\begin{abstract}
Voice disturbances are a common motor impairment seen in patients Parkinson's Disease, with voice and speech impairment estimated to effect nearly 90\% of Parkinson's Disease patients. Symptoms include reduced vocal projection (hypophonia), slow pacing of speech, and articulatory imprecision. The communication difficulties related to this hypophonia have substantial negative quality-of-life impact upon both patient and caregiver. Fortunately, there are treatment options for Parkinson's hypophonia. These span the range from behavioral interventions with speech language pathology and use of technology to
\end{abstract}


facilitate louder voicing to surgical interventions such as subthalamic nucleus deep brain stimulation and vocal fold augmentation procedures. Each of these approaches has positives and negatives, and none is universally effective. This presentation will discuss the quality-of-life impact that Parkinson's hypophonia creates and describe approaches to improving voice quality in this patient population. Focus will be placed upon the role of vocal fold augmentation, and techniques will be described to help participants consider bringing vocal fold augmentation into their Parkinson's Disease clinics, emphasizing the importance of collaborative care between Neurology and Otolaryngology teams.

\title{
Speech-Language Pathology Interventions for Voice and Swallowing in PD
}

\author{
Donna C. Tippett \\ Johns Hopkins University, Baltimore, MD, USA
}

\begin{abstract}
Hypokinetic dysarthria in Parkinson's disease is characterized by reduced vocal loudness, monoloudness, monotone, breathy and hoarse phonation, imprecise articulation, short rushes of speech, and dysfluency. These perceptual deviations can hamper functional communication and negatively impact participation in work and family life. Speech-language pathology intervention is the mainstay of behavioral therapy for hypokinetic dysarthria in Parkinson's disease. Sound therapy should embody the fundamental principles of exercise physiology, including goal selection (strength, endurance, power), specificity of training, and overload/progression. In addition, speech/voice therapy should incorporate a person-centered approach which facilitates the authentic involvement of patients and their caregivers, and addresses individual needs and circumstances. In this presentation, the ways in which speech-language pathology intervention addresses exercise physiology principles and a person-centered approach are examined. Treatment candidacy and therapy outcomes are described. Specific recommendations are provided regarding baseline speech/voice assessment, evaluation of cognition/language, patient/caregiver education, early initiation of therapy, adherence, and follow-up.
\end{abstract}

\section{Novel Approach to Early Diagnosis of Parkinson's Disease}

\author{
Michael Ugrumovi,2*, Zuleykha Zalyalova ${ }^{2}$, Razina Nigmatullina ${ }^{3}$, Alexander Kim ${ }^{1}$, Sofia Georgieva ${ }^{4}$ and Gulnara \\ Khakimova $^{1}$
}

${ }^{1}$ Institute of Developmental Biology of Russian Academy of Sciences, Russia

${ }^{2}$ Republic Clinical Diagnostic Centre of Extrapyramidal Pathology and Botulinum Therapy, Russia

${ }^{3}$ Early Diagnostics of Neurodegenerative Diseases Ltd, Kazan, Russia

${ }^{4}$ Institute of Gene Biology of Russian Academy of Sciences, Russia

\begin{abstract}
One of the priorities in Neurology is development of preclinical diagnosis of Parkinson's disease (PD), mostly by searching for biomarkers in body fluids. The weak point is that biomarkers are identified in patients at clinical stage with no guarantee of their presence at preclinical stage. The objectives of this study were: (i) search for changes in blood of drug-naïve patients at early clinical stage and MPTP-treated mice at presymptomatic and early symptomatic stages, (ii) development of a provocation test for recognition of latent failure of the nigrostriatal system in presymptomatic mice. HPLC, ELISA, Western blot, PCR-real time were used to assess changes in blood in patients and MPTP-treated mice. Moreover, we studied an action of the inhibitor of dopamine synthesis on motor behavior of mice at presymptomatic stage. It was shown that some biomarkers, detected in blood of patients were also present in mice at symptomatic stage (e.g., DOPAC, L-DOPA in plasma, D3 gene expression in lymphocytes). However, only few of them (e.g., L-DOPA, D3) were detected in presymptomatic mice, suggesting that only these markers are suitable for preclinical diagnosing. Moreover, we developed a provocation test for recognition of failure of dopaminergic system in presymptomatic mice. Inhibitor of dopamine synthesis in certain doses caused reversible depletion of striatal dopamine and motor disorders in presymptomatic mice, but not in the control. Thus, we developed a novel methodology for preclinical diagnosing of $\mathrm{PD}$, basing on a search for biomarkers in blood in patients and experimental models, and on a use of provocation test.
\end{abstract}




\title{
Identifying Park-weight Phenotype in Parkinson's Disease: Implications on Disease Management
}

\author{
Jagdish Sharma \\ Lincoln County Hospital, Lincoln, UK \\ Geriatric Neurology, University of Lincoln, Lincoln, UK
}

\begin{abstract}
A number of phenotypes are being identified in the neurodegenerative Parkinson's disease, mostly in reference to the nonmotor symptoms. One purpose of identifying phenotypes is to manage disease process more effectively. PD patients have variable impairment of olfaction; a high proportion develop body-weight change (gain or loss) as the disease advances. PD patients have a lower body weight as compared to non-PD controls. Weight loss in PD is not a benign phenomenon. Lower initial body weight and weight loss during the course of the disease predispose to the risk of dyskinesia; there being a relationship between body weight and levodopa dose per kilogram for dyskinesia. Additionally, weight loss increases the risk of under-nutrition, frailty, poor quality of life and mortality. Patients at the risk of weight loss may be identified by their severe olfactory loss (anosmia) at an early stage, since anosmia, as compared to hyposmia, seems to represent more severe neurodegenerative process predisposing to weight loss and dyskinesia describing the "olfaction-weight-dyskinesia" phenotype in Parkinson's disease. Weight loss is not due to higher energy expenditure or lower energy intake. The basis of severe neurodegenerative process and weight loss might be a longer pre-clinical phase in this phenotype. PD patients should be monitored for weight loss and the dose of levodopa adjusted accordingly as the disease advances. Measures should be taken to prevent weight loss in such patients to prevent the low body-weight related non-motor and motor adverse effects. This may result into better quality of life.
\end{abstract}

\section{$\underline{\text { Poster Presentations }}$}

\section{Mechanism of Autophagy-lysosomal Pathway Dysfunction in Gaucher Disease iPSC Neurons}

\author{
Antanina Voit BS*, Ola Awad and Ricardo Feldman \\ University of Maryland School of Medicine, Baltimore, MD, USA
}

\begin{abstract}
Gaucher disease (GD) is a lysosomal storage disorder caused by mutations in the GBA1 gene. GBA1 mutation is also a major risk factor for Parkinson's disease. GCase's deficiency results in toxic aggregations of glycolipids in cells of nervous and reticuloendothelial systems. The autophagy-lysosomal pathway (ALP) that normally degrades the aggregates is defective in GBA1 mutant neurons. The mechanism of GBA1-associated ALP dysfunction is not yet understood. Using induced pluripotent stem cells (iPSCs) from patients with GD, we investigated the mechanisms responsible for ALP dysfunction in GBA1 mutant neurons. We found that GBA1 mutation results in dysregulation of transcription factor EB (TFEB), an important regulator of lysosomal homeostasis and autophagy. TFEB levels were significantly decreased in GBA1 mutant neurons, and there was lysosomal depletion and autophagy block. Treatment of mutant neurons with recombinant GCase enzyme increased lysosomal numbers and reversed autophagy block. TFEB is regulated by the mammalian target of rapamycin complex 1 (mTORC1) at the surface of the lysosome. We hypothesized that increased neuronal sphingolipids in mutant neurons may affect mTORC1 regulation of TFEB. Our data showed that mTORC1 is hyperactive in GBA1 mutant neuronal progenitors compared to controls, both in basal conditions and following treatment with mTOR inhibitors. Our results suggest that the abnormal lipid profile in GD affects mTOR activity, which in turn alters TFEB regulation. In future experiments, we will use our GD-iPSC model to screen for compounds capable of restoring the default mTOR regulation of TFEB, which is important for developing new therapies against GBA1-associated neurodegeneration.
\end{abstract}

\section{A $\beta$ Induces Oxidative Stress in Senescence-Accelerated (SAMP8) Mice}

\author{
Kurara Takagane*, Keisuke Wakasugi and Shoichi Ishiura \\ The University of Tokyo, Japan
}




\title{
Abstract
}

Alzheimer's disease patients $(\mathrm{AD})$ accumulates amyloid $\beta(\mathrm{A} \beta)$ in their brains and $\mathrm{A} \beta$ triggers cell death and memory deficit. We developed a rice $A \beta$ vaccine expressing $A \beta$, which reduced brain $A \beta$ levels in the Tg 2576 mouse model of familial $A D$. In this presentation, we used senescence accelerated SAMP8 mice as a model of sporadic AD and investigated the relationship between $A \beta$ and oxidative stress. Insoluble $A \beta$ tended to be reduced in SAMP8 mice fed the rice A $\beta$ vaccine and 4-HNE was also reduced. We attempted to clarify the relationship between oxidative stress and $A \beta$ in vitro. Addition of $A \beta$ peptide to the culture medium resulted in an increase in 4-HNE levels in SH-SY5Y cells. Tg2576 mice, which express large amounts of A $\beta$ in their brain, also exhibited increased 4-HNE levels; this increase was inhibited by the $A \beta$ vaccine. These results indicate that $\mathrm{A} \beta$ induces oxidative stress in cultured cells and in the mouse brain.

\section{Scolopendra subspinipes mutilans Attenuates Neuroinflammation in Symptomatic hSOD1(G93A) Mice}

\author{
Seongjin Lee ${ }^{*}$, Eunjin Yang ${ }^{2}$, Bong-Keun Song ${ }^{3}$ and Sungchul Kim ${ }^{1}$ \\ ${ }^{1}$ Department of Acupuncture \& Moxibustion, Gwang-Ju Oriental Medical Hospital, Wonkwang University, Republic of Korea \\ ${ }^{2}$ Department of Medial Research, Korea Institute of Oriental Medicine, Republic of Korea \\ ${ }^{3}$ Department of Internal Medicine, Gwang -Ju Oriental Medical Hospital, Wonkwang University, Republic of Korea
}

\begin{abstract}
Neuroinflammation is a common pathological process involved in many neurodegenerative diseases, including Alzheimer's disease (AD), Parkinson's disease (PD), and ALS. Under physiological conditions, the inflammatory processes that occur in microglial cells promote innate immunity. However, under conditions of uncontrolled inflammatory stimulation, such as abnormal protein accumulation or stress, activated microglial cells and astrocytes increase the production of neurotoxic factors that induce neurodegenerative pathology. Several studies have demonstrated that the neuroinflammation caused by activated microglial cells is the hallmark pathological feature of ALS in animals and human patients. Therefore, research has targeted therapeutic interventions that can ameliorate the effects of neuroinflammation to reduce motor neuron loss and disease severity in animal models of ALS. The centipede, Scolopendra subspinipes mutilans (SSM), is utilized in traditional Chinese and Korean medicine for the treatment of a variety of diseases, such as cancer, stroke-induced hemiplegia, apoplexy, and epilepsy. Several studies have demonstrated that water-soluble SSM extracts decrease tumors and increase immune activity in tumor-bearing mice. Kim et al. reported that SSM treatment produces a significant increase in antibiotic activity against infections of the lung and intestines. However, to date, the role of SSM therapy for neurodegenerative diseases has not been investigated. Our study assessed the effects of SSM treatment at the ST36 acupoint in hSOD1 G93A transgenic mice, an animal model of ALS.
\end{abstract}

\section{Assessment of Carotid Plaque Neovascularization by Contrast-enhanced Ultrasound and High Sensitivity C-Reactive Protein Test in Patients with Acute Cerebral Infarction: A Comparative Study}

\author{
Rong $\mathrm{Xu}^{{ }^{*}}$, Xiaohua Yin ${ }^{2}$, Weixin $\mathrm{Xu}^{3}$, Lin Jin ${ }^{1}$, Min Lu${ }^{1}$ and Yingchun Wang ${ }^{1}$ \\ ${ }^{1}$ Department of Ultrasound, Jiading Central Hospital, Shanghai, People's Republic of China \\ ${ }^{2}$ Department of Radiology, Jiading Central Hospital, Shanghai, People's Republic of China \\ ${ }^{3}$ Department of Laboratory, Jiading Central Hospital, Shanghai, People's Republic of China
}

\begin{abstract}
Vulnerable carotid plaque easily ruptures and causes cerebral infarction. Plaque inflammation and neovascularization have both been shown as important characteristics in vulnerable plaque. We assessed neovascularization within carotid plaque using contrast-enhanced ultrasound, and also assessed inflammation, using high sensitivity C-reactive protein (hs-CRP) testing, in acute cerebral infarction patients. A total of 106 patients with acute cerebral infarction and 40 controls were enrolled in the study. All subjects had been previously found to have carotid atherosclerotic plaques, and the plaques were classified as soft plaque, hard plaque, mixed plaque, and calcified plaque, using carotid artery ultrasound. Contrast-enhanced ultrasound was performed on the plaques for quantitative analysis and hs-CRP levels were measured. The results showed that plaque enhancement was present in $81.1 \%$ of cerebral infarction patients and $40.0 \%$ of controls. The contrast parameters for cerebral infarction patients were significantly different from controls. For cerebral infarction patients, soft plaque showed the highest enhanced percentage, 95.1\%, with contrast parameters significantly different to other types of plaque. The hs-CRP levels of enhanced cerebral
\end{abstract}


infarction patients were higher than in non-enhanced patients. Correlation analysis in cerebral infarction patients showed that hs-CRP levels were closely related to the contrast parameters. Acute cerebral infarction patients showed intense contrast enhancement and inflammation in carotid plaque, and different types of plaque had various degrees of enhancement, suggesting that contrast-enhanced ultrasound and hs-CRP might be used for plaque risk stratification.

\title{
Therapeutic Links between Anti-cancer Drugs (Bexarotene) and Alzheimer's Disease
}

\author{
Krishna Pathak \\ Macedoniya University, Greece
}

\begin{abstract}
Background: Cancer is a disease which affects the elderly, just like Alzheimer's Disease (AD) which is also a common disease affecting older people. Also, assessing the elderly cancer affected population is a challenge in clinical practice for distinguishing between AD symptoms and cancer and its proper treatment. Most of the symptoms are related with both these diseases, therefore there may be therapeutic link between anticancer drugs and AD. Little is known anticancer drugs may help to reduce cognitive impairment, reduce $A \beta$ protein, decrease amyloid plaques and promote neuroprotection.
\end{abstract}

Objective: To synthesize the studies aimed at therapeutic links between Alzheimer's disease and anticancer drugs.

Design: To identify potential new treatment possibilities we applied the systematic searches of key electronic databases, supplemented by hand searches of reference lists. Relevant articles $\mathrm{n}$ were searched for on the following databases: PubMed, Psych Info, Medline, Scopus, Google Search engine, Cochrane library. The inclusion criteria were; (1) Studies aimed at improving cancer and anticancer therapies with human model (2) Peer-reviewed and written in English without any searching limitation on the basis of published date (3) Studies of drugs related to cancer treatment that were relevant to AD. (4) We did not include the clinical news, case report and animal trial studies.

Results: We yielded 45,366 papers. These papers were reduced after filtering titles, abstract, references published in English into one study that has been condensed into one volume of literature. There is very hard to find studies aimed at therapeutic links between Alzheimer's disease and Anti-cancer drugs in human model. However, sufficient studies are conducted with animal model (mice) and indicating the positive benefits to the $\mathrm{AD}$ patients. Bexarotene is useful for the reduction of cholesterol, to restore cognitive functions, loss of function associated with APOE $\varepsilon 4$, peripheral thyroid hormone metabolism and athyreotic and amyloid beta that is a key cofactor of Alzheimer's disease.

Conclusion: Cancer drugs are not a miracle cure for AD- dozens of questions have been raised, however, Bexarotene (chemotherapy) improves cognition and other symptoms that appear with AD patients and will be a significant drug in the coming decades.

\section{Protective Effect of Pyrroloquinoline Quinone (PQQ) in Rat Model of Intracerebral Hemorrhage}

\author{
Hongjian Lu \\ The First Affiliated Hospital of Nanjing Medical University, China
}

\begin{abstract}
Pyrroloquinoline quinone (PQQ) has invoked considerable interest because of its presence in foods, antioxidant properties, cofactor of dehydrogenase, and amine oxidase. Protective roles of PQQ in central nervous system diseases, such as experimental stroke and spinal cord injury models have been emerged. However, it is unclear whether intracerebral hemorrhage (ICH), as an acute devastating disease, can also benefit from PQQ in experimental conditions. Herein, we examined the possible effect of PQQ on neuronal functions following $\mathrm{ICH}$ in the adult rats. The results showed that rats pretreated with PQQ at $10 \mathrm{mg} / \mathrm{kg}$ effectively improved the locomotor functions, alleviated the hematoma volumes, and reduced the expansion of brain edema after ICH. Also, pretreated rats with PQQ obviously reduced the production of reactive oxygen species after ICH, probably due to its antioxidant properties. Further, we found that, Bcl-2/Bax, the important indicator of oxidative stress insult in mitochondria after ICH, exhibited increasing ratio in PQQ-pretreated groups. Moreover, activated caspase-3, the apoptotic executor, showed coincident alleviation in PQQ groups after ICH. Collectively, we speculated that PQQ might be an effective and potential neuroprotectant in clinical therapy for ICH.
\end{abstract}




\title{
Dysbindin-1, A Schizophrenia-related Protein, Interacts with HDAC3
}

\author{
Mika Soma $^{1^{*}}$, Yuichiro Watanabe ${ }^{1}$ and Shoichi Ishiura ${ }^{2}$ \\ ${ }^{1}$ The University of Tokyo, Japan \\ ${ }^{2}$ Doshisha University, Japan
}

\begin{abstract}
Dystrobrevin-binding protein 1 (DTNBP1) is one of the top candidates genes in schizophrenia. It has several SNPs and these SNPs are reported to be associated with schizophrenia. DTNBP1 encodes dysbindin-1, which is associated with working memory capacity in normal subjects. Dysbindin- 1 has three isoforms - A, B and C, all of which have been reported to decrease in the brain of schizophrenia patients, and dysbindin-1 mutant animals showed similar patterns to schizophrenia. Histone Deacetylase 3 (HDAC3) is one of the members of the histone deacetylase super family, which represses transcription in nucleus. HDAC3 has been reported to repress memory formation. HDAC inhibitors have a long history of use in psychiatric disorders including schizophrenia. Several studies have shown that both dysbindin-1 and HDAC3 can be phosphorylated by the DNAdependent protein kinase complex. So we investigated the functional relationship between dysbindin-1 and HDAC3.

Here we report the identification of a previously unrecognized molecular pathway involving a protein-protein interaction of dysbindin-1 and HDAC3. The interaction between dysbindin-1 and HDAC3 occurred in an isoform-specific manner: HDAC 3 coupled with dysbindin-1A and $-1 B$, but not $-1 \mathrm{C}$. We also found that dysbindin-1B expression was increased in the nucleus in the presence of HDAC3, and, conversely, that the phosphorylation level of HDAC3 increased in the presence of dysbindin-1B. Our results may provide a novel target in the treatment of neuropathological disorders such as schizophrenia.
\end{abstract}

\section{Assessment of Glutathione System and its Related Enzymes in the Serum of Children with Autistic Spectrum Disorders}

\author{
Azouz HG*, Bedair HA, Kitat AF and Abdelkarim BM \\ Pediatric Neurology Department and Biochemistry Department, Faculty of Medicine, Alexandria University, Egypt
}

\begin{abstract}
Introduction: Autistic Spectrum Disorders (ASD) are a group of neurodevelopmental conditions characterized by impairment in social communication/ social interaction, and repetitive or restricted interests or behaviors. Increasing evidence suggests a role of oxidative stress in the development and clinical manifestation of autism. It is suggested that autism may result from an interaction between genetic, environmental, and immunological factors, with oxidative stress as a mechanism linking these risk factors.
\end{abstract}

Aim of Study: This study aimed at assessing glutathione system and its related enzymes in children with autistic spectrum disorders as an indication of oxidative stress.

Subjects and Methods: Thirty ASD children with age ranging from three to ten years were recruited from patients attending neurobehavioral outpatient clinic at the Alexandria University Children's Hospital. Thirty healthy child served as control. All subjects were evaluated clinically, and serum reduced glutathione, glutathione reductase activity, glutathione peroxidase activity, glutathione s-transferase activity and serum selenium were measured. Cases only were evaluated by DSM-V and childhood Autism rating scale (CARS).

Results: Autistic children had low level of reduced glutathione, low activity of glutathione reductase enzyme, glutathione s-transferase enzyme and serum selenium level compared to controls with a statistically significant difference between both groups $(\mathrm{P}=0.031,<0.001,<0.001,<0.001$ respectively). There was significant correlation between selenium level and CARS, where cases with low level of selenium had high score of CARS. There was a significant relation between bowel dysfunction and abdominal cramps with reduced glutathione level ( $p=0.033,0.009$ respectively). Sleep disorders showed significant relation with reduced glutathione level and glutathione $s$-transferase activity ( $p$ value $=0.007,0.010$ respectively) while self-injurious behavior showed a significant relation with glutathione reductase $(\mathrm{p}=0.038)$.

Conclusion: The current study lends further support to the concept that an increased vulnerability to oxidative stress may contribute to the development of manifestation of Autism. Potential treatment protocols should be evaluated to potentially correct the oxidative stress abnormalities observed in those children. 


\title{
The Purinergic Receptor P2X7: A Potential New'Therapeutic Target in Bipolar Disorder
}

\author{
Carolina Gubert ${ }^{*}$, Roberta Andrejew ${ }^{1}$, Robson Coutinho-Silva ${ }^{3}$, Fernanda Bueno Morrone ${ }^{4}$, Flávio Kapczinski ${ }^{2}$, Pedro \\ Magalhães ${ }^{2}$ and Ana Maria Oliveira Battastini' ${ }^{1}$ \\ ${ }^{1}$ Graduate Program in Biological Science: Biochemistry, Universidade Federal do Rio Grande do Sul, Brazil \\ ${ }^{2}$ Bipolar Disorder Program and Laboratory of Molecular Psychiatry, Hospital de Clinicas de Porto Alegre, Brazil \\ ${ }^{3}$ Universidade Federal do Rio de Janeiro, Brazil \\ ${ }^{4}$ Pharmacy Faculty, Pontifícia Universidade Católica do Rio Grande do Sul, Brazil
}

\begin{abstract}
The precise mechanisms underlying Bipolar Disorder (BD) pathophysiology remains unknown. Evidence points to an increase of neuroinflammation and excitotoxicity. The purinergic receptor P2X7 (P2X7R) has been directly implicated to these parameters. In the present work, we aimed to explore the participation of the P2X7R on behavioral, inflammatory and neurotransmitters response in a pharmacological animal model of mania. The results are summarized as follows: the animal pharmacological model of mania significantly increased locomotor activity, mean speed and number of rearings in mice and the antagonist of P2X7R significantly reduce all the behavioral parameters. The animal model of mania had no behavioral effects in P2X7R-/- mice when compared to controls. There was a significant increase in IL-1 $\beta$ and TNF- $\alpha$ levels in striatum and hippocampus after the animal model, which was reversed by the treatment with the antagonist of P2X7R. The dopamine metabolite DOPAC is increased in the animal model and the antagonist of P2X7R significantly revert it. Our results suggest that the P2X7R participates in the establishment of the animal model of mania, modulated by the proinflammatory and neurotransmitter environment and ultimately affecting the behavioral changes. In conclusion, our findings suggest that P2X7R has the potential to become a new therapeutic target in BD.
\end{abstract}

\section{Prediction of Neurodevelopmental Outcome of Preterm Babies ( $\leq 33$ Weeks) Using Risk Stratification Score}

\author{
Radhika $\mathrm{S}^{1^{*}}$ and Naveen Jain ${ }^{2}$ \\ ${ }^{1}$ Government Medical College, Thiruvananthapuram, Kerala, India \\ ${ }^{2}$ Kerala Institute of Medical Sciences, Thiruvananthapuram, Kerala, India
}

\begin{abstract}
Objectives: To develop a score based on perinatal risk factors to predict major neurodevelopmental disability (NDD) at one year of age in a cohort of preterm Indian babies ( $\leq 33$ weeks).

Methods: Prospective analytic study at a referral neonatal intensive care unit (NICU) with a developmental follow up clinic. All preterm babies born < 33 weeks, discharged from the NICU were included. Pre-defined perinatal and neonatal risk factors were recorded. Babies were followed to one year of age, corrected for prematurity, for major NDD (defined as cerebral palsy or development quotient by Development assessment scale for Indian Infants (DASII) motor /mental score < 70 or blindness in one or both eyes or hearing impairment needing hearing aids).
\end{abstract}

Results: The incidence of major NDD among the 225 babies was 6.2\%. A clinical score was devised by combining 5 risk factors, gestation $\leq 28$ weeks, need for extensive resuscitation at birth, symptomatic hypoglycemia, invasive ventilation for $>7$ days and abnormal neurosonogram; each item scored as 0 or 1 based on absence or presence of the risk factor. Scores of 1 to 5 were associated with $4 \%, 6 \%, 10 \%, 25 \%, 100 \%$ risk of major NDD respectively $(\mathrm{p}<0.01)$. We could stratify $87.5 \%$ of the babies into low risk (score of 1,2) and 12.5\% into high risk (score 3, 4 or 5) for major NDD. The incidence of major NDD was only $4.5 \%$ in the low risk group as compared to $17.8 \%$ among high risk babies.

Conclusion: Majority of the preterm babies at lower risk for NDD need less intensive follow up, while those at higher risk should be guided to more frequent structured follow up and early intervention program. 


\title{
Levetiracetam Prophylaxis Ameliorates Seizure Epileptogenesis after Fluid Percussion Injury
}

\author{
Yuan-Hao Chen ${ }^{1^{*}}$, Eagle Yi-Kung Huang ${ }^{2}$, Tung-Tai Kuo ${ }^{3}$, Barry J. Hoffer ${ }^{4}$, Hsin-I Ma1 and Yung-Hsiao Chiang ${ }^{5}$ \\ ${ }^{1}$ Department of Neurological Surgery, Tri-Service General Hospital, National Defense Medical Center, Taipei, Taiwan \\ ${ }^{2}$ Department of Pharmacology, National Defense Medical Center, Taipei, Taiwan \\ ${ }^{3}$ Graduate Institute of Computer and Communication Engineering, National Taipei University of Technology, Taipei, Taiwan \\ ${ }^{4}$ Department of Neurosurgery, Case Western Reserve University School of Medicine, Cleveland, OH, USA \\ ${ }^{5}$ Graduate Program on Neuroregeneration, Taipei Medical University, Taipei, Taiwan
}

\begin{abstract}
To determine whether post-traumatic seizure severity would be affected by the interval between seizures and head injury, we measured seizures after various times with or without fluid percussion brain injury (2atm fluid percussion injury; FPI). To determine efficacy of anti-seizure medication, we also determined if levetiracetam (LEV) would alter the relationship between injury and subsequent seizures. Early post-traumatic seizures were induced by Kainic acid (KA) at one week after 2atm fluid percussion injury (FPI) in one group (FPI-ES). Seizures were induced at two weeks after FPI by KA in another group (FPILS). In addition, one group had induced seizures by KA without FPI, (sham-ES). Finally, one group of animals received the antiepileptic agent (levetiracetam) infusion for one week after FPI and then had seizures induced by KA (FPI-LEV-ES). We measured seizure onset time, ictal duration and severity of seizures using a modified Racine's scale. Synaptic plasticity in each group was analyzed and histopathological changes in the hippocampus CA1 region were also analyzed. Severity of seizures were increased in the FPI-ES group compared with sham-ES animals. Severity was also enhanced in early post-injury seizures induced by KA (FPI-ES vs. FPI-LS); this exacerbation of seizure severity could be ameliorated by levetiracetam infusion (FPI-ES vs. FPI-LEV-ES). Long term potentiation (LTP) in the Schaffer collateral pathway was suppressed in the FPI-ES group and this was partially reversed by LEV. Neuronal degeneration in CA1 was more severe in the FPI-ES group and this degeneration was also diminished by LEV. We conclude that early post injury seizures exacerbate susceptibility and severity of post traumatic seizures and increase neuronal degeneration in the CA1 layer of hippocampus, together with suppression of LTP in the Schaffer collateral pathway. These are partially reversed by LEV infusion after FPI.
\end{abstract}

\section{Estimation of the Impact of a Japanese Encephalitis Immunization Program with Live, Attenuated SA 14-14-2 Vaccine in Nepal}

\author{
Shyam Raj Upreti', Kristen Janusz, W. William Schluter, Ram Padarath Bichha, Geeta Shakya Brad J. Biggerstaff, Murari \\ Man Shrestha, Tika Ram Sedai, Marc Fischer, Robert V. Gibbons, Sanjaya Shrestha and Susan L. Hills
}

Group of Technical Assistance (GTA), Nepal

\begin{abstract}
Background: JE has been recognized as a public health problem in Nepal since the mid-1970s. Wider availability of the live, attenuated SA 14-14-2 Japanese encephalitis (JE) vaccine has facilitated introduction or expansion of immunization programs in many countries. However, information on their impact is limited. In 2006, Nepal launched a JE immunization program and by 2009 mass campaigns had been implemented in 23 districts.
\end{abstract}

Methods: To describe the impact, we analyzed surveillance data from 2004 through 2009 on laboratory-confirmed JE and clinical acute encephalitis syndrome (AES) cases.

Findings: The post-campaign JE incidence rate of 1.3 per 100,000 populations was $72 \%$ lower than expected had no campaigns occurred and an estimated $891 \mathrm{JE}$ cases were prevented. In addition, AES incidence was 58\% lower, with an estimated 2787 AES cases prevented, suggesting that three times as many disease cases may have been prevented than indicated by the laboratory-confirmed JE cases alone. These results provide useful information on preventable JE disease burden, and the potential value of JE immunization programs. 


\title{
Melatonin Maintains Mitochondrial Homeostasis and Promotes Peuroprotection by Inducing Autophagy Against Oxaliplatin-evoked Peripheral Neuropathy
}

\author{
Aparna A*, Prashanth K and Ashutosh Kumar \\ Department of Pharmacology E Toxicology, National Institute of Pharmaceutical Education and Research, NIPER-Hyderabad, \\ Telangana, India
}

\begin{abstract}
Oxaliplatin, an organoplatinum compound used in the treatment of colorectal cancer, but its use is often limited due to the severe incidence of peripheral neuropathy. Mitochondrial dysfunction has been implicated as a major patho-mechanism in the oxaliplatin induced neurotoxicity. Considering melatonin as an antioxidant and mitoprotectant, the current study delineated its neuroprotective role against oxaliplatin induced peripheral neuropathy (OIPN). Melatonin (at $15 \& 30 \mu$ mol) reduced the mitochondrial superoxide, prevented the loss of mitochondrial membrane potential $(\Psi \mathrm{m})$ and promoted the neuritogenesis in the oxaliplatin (at $50 \mu \mathrm{mol}$ ) insulted neuro-2a cells. It did not alter the anticancer activity of oxaliplatin in human colon cancer cell line, HT-29. Melatonin (at $3 \& 10 \mathrm{mg} / \mathrm{kg}$ i.p.) treatment significantly (at high dose $\mathrm{p}<0.001$ ) alleviated the oxaliplatin ( $4 \mathrm{mg} / \mathrm{kg}$ twice weekly for 4 weeks, i.p.) induced pain behaviour along with the functional defects in rats. Melatonin efficiently ameliorated the oxaliplatin induced nitro-oxidative stress, loss of $\Psi \mathrm{m}$, increased levels of mitochondrial superoxide dismutase and improved the mitochondrial complexes function and ATP levels in vivo. Melatonin's protective effects were attributed to its role in preventing the oxaliplatin induced apoptosis (TUNEL assay) and increasing autophagy pathway (via LC3A/B) in peripheral nerve and dorsal root ganglion (DRG) which was further confirmed through immunohistochemical and western blotting analysis. Hence, maintained the mitochondrial function and cellular bioenergetics. Taken together, our results demonstrated an array of evidence for the neuroprotective potential of melatonin and it can be proposed as a possible candidate in the management of neuropathic pain.
\end{abstract}

\section{Differentiating Between Subtypes of Primary Progressive Aphasia and Mild Cognitive Impairment on a Modified Version of the Frontal Behavioral Inventory}

\author{
Donna C. Tippett ${ }^{1-3^{*}}$, Cornelia Demsky ${ }^{1}$, Rajani Sebastian ${ }^{1}$, Amy Wright $^{1}$, Cameron Davis $^{1}$ and Argye E. Hillis $^{1,2,4}$ \\ ${ }^{1}$ Department of Neurology, Johns Hopkins University School of Medicine, Baltimore, MD, USA \\ ${ }^{2}$ Department of Physical Medicine and Rehabilitation, Johns Hopkins University School of Medicine, Baltimore, MD, USA \\ ${ }^{3}$ Department of Otolaryngology-Head and Neck Surgery, Johns Hopkins University School of Medicine, Baltimore, MD, USA \\ ${ }^{4}$ Department of Cognitive Science, Krieger School of Arts and Sciences, Johns Hopkins University, Baltimore, MD, USA
}

\begin{abstract}
We explored the ability of a modified version of the Frontal Behavioral Inventory (FBI-mod) to discriminate between patients with distinct subtypes of primary progressive aphasia (PPA) and patients with mild cognitive impairment (MCI). We hypothesized that individuals with nonfluent agrammatic PPA (nfaPPA) would have higher negative behavior scores than other groups and that individuals with semantic variant PPA (svPPA) would have higher disinhibition scores than other groups. Family members and/or caregivers of 87 individuals with PPA and MCI completed the FBI-mod (logopenic PPA, lvPPA $\mathrm{n}=29$; mean age $70.55+6.73$; nfaPPA $n=20$, mean age $66.65+10.94$; svPPA $n=15$, mean age $67.60+5.74$; unclassifiable PPA, UnCPPA $n=7$, mean age $70.29+7.06$, MCI $n=16$; mean age $69.0+12.09$ ). The groups were not significantly different in age, education, or disease duration. There were no significant differences between the groups on ANOVA for negative behaviors $(\mathrm{p}=0.156, \mathrm{~F}=1.71)$, although the svPPA and UnCPPA had the highest scores on this portion of the FBI-mod and individuals with MCI had the lowest score. The disinhibition scores were significantly different $(p=0.022, F=3.02)$, with svPPA having the highest score, however post hoc analysis did not reveal significant differences between group means. The odds of having svPPA was significantly higher (OR 1.04 [1.01-1.09]) for every 1 point on the disinhibition score (but not negative behavior score; ns), independent of age, disease duration, and gender $(\mathrm{p}=0.012)$. The frequency of "severe" or " 3 " ratings revealed different negative behavioral profiles for each group which may have clinical and practical implications.
\end{abstract}




\title{
Measurement of Autonomic Response to Infused Medications for Chronic Migraine as Measured by Near Infrared Spectroscopy
}

\author{
Ardy Wong*, Daryl Omire-Mayor, Pooja KA and Kambiz Pourrezaei \\ School of Biomedical Engineering, Science and Health Systems, Drexel University, 3141 Chestnut St, Philadelphia, PA, USA
}

\begin{abstract}
Pain is a phenomenon that most people experience at some point in their lives, whether minor and resulting from an injury, a symptom of a disease, or occurring chronically post initial injury or disease, making pain the problem itself - most commonly classified as a neurological disorder. Previous neuroimaging studies have been able to identify ways of quantifying pain objectively, but failing in affordability and feasibility of use in a clinical setting. Functional near infrared spectroscopy has been used as a method to objectively measure the autonomic response to noxious stimuli and has proven to be linked to subjective responses; however, this has yet to be further explored through appropriate analysis of quantifiable subjective responses. Functional near infrared spectroscopy (fNIRS) is a fairly inexpensive and portable optical brain imaging modality that uses near infrared light to track changes in cerebral hemodynamic responses. In our lab, we utilized fNIRS to compare the hemodynamic response of migraine patients $(n=41)$ receiving one of three medications: magnesium sulfate, valproate sodium, and dihydroergotamine (DHE) [1] in order to study the role of the vaso-reactivity of these drugs. The autonomic response was found to be correlated with patient's self-report pain score reduction. We have also used fNIRS to study healthy subjects' pain tolerance and adaptation to cold noxious stimuli. This study might be useful to predict subject risk factor for developing post operation chronic pain.
\end{abstract}

Citation: Proceedings of the Second Neurological Disorders Summit (NDS-2016). J Neurol Exp Neurosci 2(Suppl 2): S1-S38.

Copyright: This is an Open Access article distributed under the terms of the Creative Commons Attribution 4.0 International License (CC-BY) (http://creativecommons.org/licenses/by/4.0/) which permits commercial use, including reproduction, adaptation, and distribution of the article provided the original author and source are credited. Published by United Scientific Group.

Received: September 15, 2016 Accepted: October 19, 2016 Published: October 21, 2016 